\title{
Higher-order Properties of Approximate Estimators
}

\author{
Dennis Kristensen* $\quad$ Bernard Salanié $^{\dagger}$
}

MAY 18, 2016

\begin{abstract}
Many modern estimation methods in econometrics approximate an objective function, for instance, through simulation or discretization. These approximations typically affect both bias and variance of the resulting estimator. We first provide a higher-order expansion of such "approximate" estimators that takes into account the errors due to the use of approximations. We show how a Newton-Raphson adjustment can reduce the impact of approximations. Then we use our expansions to develop inferential tools that take into account approximation errors: we propose adjustments of the approximate estimator that remove its first-order bias and adjust its standard errors. These corrections apply to a class of approximate estimators that includes all known simulation-based procedures. A Monte Carlo simulation on the mixed logit model shows that our proposed adjusments can yield significant improvements at a low computational cost.
\end{abstract}

\section{Introduction}

The complexity of econometric models has grown steadily over the past three decades. The increase in computer power contributed to this development in various ways, and in particular by allowing econometricians to estimate more complicated models using methods that rely on approximations. Examples include simulated method of moments (McFadden (1989); Pakes and Pollard (1989); Duffie and Singleton (1993); Creel and Kristensen (2012)), simulated maximum likelihood (Lee (1992, 1995); Fermanian and Salanié (2004); Kristensen and Shin (2012)), and approximate solutions to structural models (Rust (1987); Tauchen and Hussey (1991); Fernández-Villaverde, Rubio-Ramirez and Santos (2006); Norets (2012); Kristensen and Schjerning (2015)). In all of these cases, the objective function defining the estimator includes a component which is approximated using some type of numerical algorithm. We will refer to this component as the approximator, and call the resulting estimator an approximate estimator. Taking the approximation error to zero defines an infeasible estimator which we

${ }^{*}$ UCL, IFS and CREATES. E-mail: d.kristensen@ucl.ac.uk.

${ }^{\dagger}$ Columbia University. E-mail: bsalanie@columbia.edu 
call the exact estimator. In simulation-based inference, for instance, the exact estimator would be obtained with an infinite number of simulations. In dynamic programming models solved by discretization the exact estimator would rely on an infinitely fine grid.

The use of approximations usually deteriorates the properties of the approximate estimator relative to those of the corresponding exact estimator: the former may suffer from additional biases and/or variances compared to the latter. When the approximation error is non-stochastic, its main effect is to impart additional bias to the estimator. On the other hand, stochastic approximations not only create bias; they may also reduce efficiency. The effect of the approximation on the estimator can usually be reduced by choosing a sufficiently fine approximation; but this comes at the cost of increased computation time. In many applications this may be a seriously limiting factor; increased computer power helps, but it also motivates researchers to work on more complex models. It is therefore important to quantify the additional estimation errors that approximators generate, and also to account for these additional errors in order to draw correct inference.

As a first step in this direction, we analyze the higher-order properties of the approximate estimator in a general setting. These expansions apply to a very large class of models, and they can be used to develop a number of adjustments to estimators and/or standard errors that open the way to better inference. To show this, we develop analytical bias and variance adjustments for a large class of approximate estimators where the approximation is stochastic. In simulation-based inference for instance, these adjustments remove the leading terms due to simulations. We also propose a very generally applicable two-step method; it consists of updating the approximate estimator obtained by one or several Newton-Raphson iterations based on the same objective function, but with a much finer degree of approximation. These different methods can of course be combined when they both apply.

Our theoretical results applies to generalized method of moment estimators as well as M-estimators, both when the approximation is stochastic and when it is not. The results encompass and extend results in the literature on simulation-based estimators. Moreover, the expansion can be used to analyze the behavior of estimators that rely on numerical solutions to structural dynamic models as cited above. Our results also apply to many estimators used in empirical IO, which combine simulation and numerical approximation. And it also covers situations where numerical derivatives are used, either for computation of variance estimators or optimization algorithms based on Newton iterations ${ }^{1}$. To the best of our knowledge, this is the first paper to provide results for such a general class of models.

To test the practical performance of our proposed adjustment methods, we run a simulation study on a mixed logit model. The mixed logit is one of the basic building blocks in much work in demand analysis, for instance; and it is simple enough that we can compute the

\footnotetext{
${ }^{1}$ However, in most of our examples, we abstract away from issues with numerical maximization that sometimes arise when computing extremum estimators.
} 
true value of the biases and efficiency losses, as well as our estimated corrections. We show that uncorrected SML has non-negligible bias, even for large sample sizes; and that standard confidence intervals can be wildly off the mark. Our analytical adjustment removes most of the bias at almost no additional computational cost; and it yields very reliable confidence intervals. The Newton-Raphson correction also reduces the bias and improves confidence intervals, but it does so less effectively than the analytical adjustment.

In a recent paper, Freyberger (2015) derived analytical adjustments for the Berry-LevinsohnPakes (1995) model when the numbers of consumers and/or the number of simulation draws are finite. His approach is similar to ours: his results are less general, but since he only deals with a specific model his assumptions are more primitive and his formulæ more explicit. We complement his work by providing the formulæ for our Newton-Raphson adjustment for this model in section 6.2 .

The paper is organized as follows. Section 2 presents our framework and some examples. In Section 3, we derive a higher-order expansion of the approximate estimator relative to the exact one. We describe our Newton-Raphson correction in section 4. Then in Section 5 we build on the expansion to propose adjusted estimators, standard errors, and confidence intervals. Section 6 applies the general theory to two specific approximate estimators, while Section 7 presents the results of a Monte Carlo simulation study using the simulated MLE of the mixed logit model as an example. We discuss possible extensions of our results in Section 8. Appendix A and B contain proofs of the main results and lemmas, respectively. Appendix C provides details for two examples of our theory, and Appendix D outlines how the theory can be generalized to handle multiple approximators with different properties.

\section{Framework}

Given a sample $\mathcal{Z}_{n}=\left\{z_{1}, \ldots, z_{n}\right\}$ of $n$ observations, our aim is to estimate a parameter $\theta_{0} \in \Theta \subseteq \mathbb{R}^{k}$ through an estimating equation that the "exact" estimator $\hat{\theta}_{n}$ is set to solve,

$$
G_{n}\left(\hat{\theta}_{n}, \gamma_{0}\right)=o_{P}(1 / \sqrt{n}), \text { where } G_{n}(\theta, \gamma)=\frac{1}{n} \sum_{i=1}^{n} g\left(z_{i} ; \theta, \gamma\right)
$$

and $g(z ; \theta, \gamma)$ is a known functional that depends on data, $z$, the parameter of interest, $\theta$, and a nuisance parameter $\gamma$. The nuisance parameter $\gamma$ could be finite-dimensional, but in most situations it is a parameter dependent function, $u \mapsto \gamma(u ; \theta)$. The nature of the argument $u$ of the function $\gamma$ will depend on the application; it could be covariates relative to one observation, the value of a conditional moment, or more complex objects. This is irrelevant for our general theory.

Suppose that the object $\gamma$ is not known in closed form to the econometrician, so that

the estimator $\hat{\theta}$ is infeasible. Instead, we approximate $\gamma$ by a $\hat{\gamma}_{S}$ that depends on some 
approximation scheme of order $S$ (e.g. $S$ simulations, or a discretization on a grid of size $S$ ), and compute the corresponding "approximate" estimator $\hat{\theta}_{n, S}$ satisfying

$$
G_{n}\left(\hat{\theta}_{n, S}, \hat{\gamma}_{S}\right)=o_{P}(1 / \sqrt{n})
$$

Our first aim is to analyze the impact of approximations: How do they impact the distribution of $\hat{\theta}_{n, S}$ ? This analysis is in turn used to propose methods that reduce the biases and variances due to approximations, and adjust standard errors to take into account additional noise due to approximations.

We restrict attention throughout to the case of smooth approximators where $\hat{\gamma}_{S}$ is, as a minimum, differentiable. Moreover, while $\gamma$ may be a vector-valued function, we will in the main text assume that the biases and variances due to approximations of the different components vanish at the same rate. This is merely to save on notation, and Appendix D provides results for the case of multiple approximators with possibly different rates.

We now present a few examples that fall within the above setting:

Example 1: Approximate M-estimators. Consider an M-estimator $\hat{\theta}=\arg \max _{\theta \in \Theta} Q_{n}(\theta, \gamma)$, where $Q_{n}(\theta, \gamma)=\sum_{i=1}^{n} q\left(z_{i} ; \theta, \hat{\gamma}_{S}\right) / n$. In this case, we set $g(z ; \theta, \gamma)=\partial q(z ; \theta, \gamma) /(\partial \theta)$. This covers simulated maximum likelihood estimator (SMLE) where $q(z ; \theta, \gamma)=\log \gamma(z ; \theta)$ and $\gamma$ is a density that is computed by simulations. It also includes simulated pseudomaximum likelihood (Laroque and Salanié, 1989) where $q(z, \gamma ; \theta)=-(y-\gamma(x ; \theta))^{2}$ and $\gamma(x ; \theta)=E[y \mid x ; \theta]$ is a conditional moment which is computed by simulations.

Example 2: Approximate GMM-estimators. Suppose that $\hat{\theta}$ is defined as in Example 1, but now $Q_{n}(\theta, \gamma)=M_{n}(\theta, \gamma)^{\prime} W_{n} M_{n}(\theta, \gamma)$ where $M_{n}(\theta, \gamma)=\sum_{i=1}^{n} m\left(z_{i}, \gamma ; \theta\right) / n$ is a set of sample moments and $W_{n} \stackrel{P}{\rightarrow} W>0$. Then we set $g\left(z_{i} ; \theta, \gamma\right)=H(\theta, \gamma) W m\left(z_{i} ; \theta, \gamma\right)$ where $H(\theta, \gamma)=E\left[\partial m\left(z_{i} ; \theta, \gamma\right) /(\partial \theta)\right]$. This includes simulated method of moments (SMM), where $m(z, \gamma ; \theta)=m(y)-\gamma(x ; \theta)$ and $\gamma(x ; \theta)=E[m(y) \mid x ; \theta]$, and indirect inference (Gourieroux and Monfort, 1996) where the estimator of the auxiliary model's parameters, $\beta$, can be expressed as $\hat{\beta}=\beta\left(\theta_{0}\right)+\sum_{i=1}^{n} m\left(z_{i}\right) / n+o_{P}\left(n^{-1 / 2}\right)$ and $\gamma(\theta)=E[\hat{\beta} \mid \theta]=$ $\beta(\theta)+E\left[m\left(z_{i}\right) \mid \theta\right]+o\left(n^{-1 / 2}\right)$.

Example 3: Estimation of dynamic structural models. Examples 1-2 also cover MLE and GMM estimators of structural models, where $\gamma$ is the value function of a dynamic programme. In Discrete Choice Programming Models, simulations are combined with discretization or sieve methods (parametric approximations) to approximate the value function; see Rust (1987), Keane and Wolpin (1994, 1997), Norets (2012), and Kristensen and Schjerning (2015). Similarly, many models used in macroeconomics are so complex that estimation is based on an approximate density (model), which is often obtained by so-called pertubation or projection methods; see Judd, Kubler and Schmedders (2003), Fernández-Villaverde, Rubio-Ramirez and Santos (2006) and Ackerberg, Geweke and Hahn (2009). 
Example 4: Numerical inversion and derivatives. Some estimators involve numerical inversion of a function. One example of this is the estimator of discrete choice models proposed in Berry, Levinsohn and Pakes (1995) which combines numerical inversion of a simulated version of the so-called market share function. Here, $\gamma$ is the inverse of the simulated market share function. See also Judd and $\mathrm{Su}$ (2012) and Dubé, Fox and Su (2012) and Freyberger (2015) for variations over and more results on this procedure. Similarly, derivatives of the sample objective function are often approximated numerically, either to maximize it or to estimate the asymptotic variance, e.g. $\sum_{i=1}^{n} \gamma_{k}\left(z_{i} ; \theta\right) / n$, where $\gamma_{k}(z ; \theta)=\partial q\left(z_{i} ; \theta\right) /\left(\partial \theta_{k}\right)$ for $k=1, \ldots, \operatorname{dim}(\theta)$. We replace $\gamma_{k}(z ; \theta)$ with, for example, $\hat{\gamma}_{S, k}(z ; \theta)=\left[q\left(z_{i} ; \theta+\epsilon_{S} e_{k}\right)-q\left(z_{i} ; \theta-\epsilon_{S} e_{k}\right)\right] /\left(2 \epsilon_{S}\right)$, where $e_{k}$ is the $k$ th column of the identity matrix and $\epsilon_{S} \rightarrow 0$ as $S \rightarrow \infty$. Our theory applies to approximate variance estimators built around numerical derivatives, as well as to estimators built around quasi-Newton iterations that use numerical derivatives; see also Hong, Mahajan and Nekipelov (2015) and Bruyns et al (2015).

\subsection{Estimating Equation}

To analyze the impact of approximations, we assume that the function of interest $\gamma_{0}: \mathcal{U} \times \Theta \mapsto$ $\mathbb{R}^{p}$ belongs to a linear function space $\Gamma$ equipped with a norm $\|\cdot\|$. In most cases, the norm will be either the sup-norm, $\|\gamma\|=\sup _{u \in \mathcal{U}} \sqrt{\left(\gamma(u)^{\prime} \gamma(u)\right)}$, or some $L^{q}$-norm induced by the probability measure associated with the data generating process, $\|\gamma\|=E\left[\left(\gamma(u)^{\prime} \gamma(u)\right)^{q / 2}\right]^{1 / q}$ for some $q \geq 1$. Our analysis will involve the following sample and population averages,

$$
H_{n}(\theta, \gamma)=\frac{1}{n} \sum_{i=1}^{n} h\left(z_{i} ; \theta, \gamma\right), \quad G(\theta, \gamma)=E\left[g\left(z_{i} ; \theta, \gamma\right)\right], \quad H(\theta, \gamma)=E\left[h\left(z_{i} ; \theta, \gamma\right)\right]
$$

where $h\left(z_{i} ; \theta, \gamma\right)=\partial g\left(z_{i} ; \theta, \gamma\right) /(\partial \theta)$. We first impose conditions to ensure that the exact, but infeasible estimator is well-behaved:

A.1 (i) $\hat{\theta}_{n} \stackrel{P}{\rightarrow} \theta_{0}$ which lies in the interior of the parameter space $\Theta$; (ii) $\left\{z_{i}\right\}$ is stationary and geometrically $\alpha$-mixing; (iii) $E\left[\left\|g\left(z_{i} \gamma_{0}\right)\right\|^{2+\delta}\right]<\infty$ for some $\delta>0$; (iv) $G\left(\theta_{0}, \gamma_{0}\right)=0$.

A.2 (i) $H_{0}:=H\left(\theta_{0}, \gamma_{0}\right)$ is positive definite; (ii) for some $\delta>0, E\left[\sup _{\left\|\theta-\theta_{0}\right\|<\delta}\left\|h\left(z_{i} ; \theta, \gamma_{0}\right)\right\|\right]<$ $\infty$; (iii) $E\left[\sup _{\left\|\theta-\theta_{0}\right\|<\delta}\left\|h\left(z_{i} ; \theta, \gamma\right)-h\left(z_{i} ; \theta, \gamma_{0}\right)\right\|\right] \leq \bar{H}\left\|\gamma-\gamma_{0}\right\|^{\lambda}$ for some $\delta, \lambda, \bar{H}>0$ and neighbourhood $\mathcal{N}$ of $\gamma_{0}$, and all $\gamma \in \mathcal{N}$.

Assumption A.1(i) requires that the infeasible estimator be consistent; Lemma 1 below provides a set of sufficient conditions. A.1(ii) rules out strongly persistent data, thereby allowing us to obtain standard rates of convergence for the resulting estimators. In particular, A1(ii) and A.1(iii) together imply that a central limit theorem (CLT) applies to $G_{n}\left(\theta_{0}, \gamma_{0}\right)$. 
The geometric mixing condition could be weakened, but would complicate the analysis; see Kristensen and Shin (2012) for some results in this direction. Assumption A.2 imposes differentiability of $\theta \mapsto g(z ; \theta, \gamma)$. In particular, when $\gamma$ depends on $\theta$ (as is the case for all of our examples), it requires that the approximator be a smooth function of $\theta$. Therefore A.2 rules out discontinuous and non-differentiable approximators, such as the simulated method of moment estimators for discrete choice models proposed in McFadden (1989) and Pakes and Pollard (1989) which involve indicator functions. ${ }^{2}$ The Lipschitz condition imposed on $h(z ; \theta, \gamma)$ is used to ensure that $H_{n}\left(\theta, \hat{\gamma}_{S}\right) \stackrel{P}{\rightarrow} H(\theta, \gamma)$ uniformly in $\theta$ as $\hat{\gamma}_{S} \stackrel{P}{\rightarrow} \gamma$.

Since our focus is on higher-order properties of the approximate estimator, we also assume consistency of this so that we can conduct our analysis locally around $\theta_{0}$ :

A.3 $\hat{\theta}_{n, S} \stackrel{P}{\rightarrow} \theta_{0}$ as $n, S \rightarrow \infty$.

A set of sufficient conditions for Assumptions A.1 (i) and A.3 to hold are provided in the following lemma, the proof of which simply involves verifying the conditions of Newey and McFadden (1994, Theorem 2.1) and so is left out.

Lemma 1 Suppose that $\hat{\theta}_{n, S}=\arg \max _{\theta \in \Theta} Q_{n}\left(\theta, \hat{\gamma}_{S}\right)$ where: (i) $\Theta$ is compact; (ii) $\hat{\gamma}_{S} \stackrel{P}{\rightarrow} \gamma_{0}$; (iii) either $\sup _{\theta \in \Theta,\left\|\gamma-\gamma_{0}\right\|<\delta}\left|Q_{n}(\theta, \gamma)-Q(\theta, \gamma)\right| \stackrel{P}{\rightarrow} 0$ or $\left|Q_{n}\left(\theta, \gamma_{1}\right)-Q_{n}\left(\theta, \gamma_{2}\right)\right| \leq B_{n}\left\|\gamma_{1}-\gamma_{2}\right\|$ for all $\gamma_{1}, \gamma_{2}$ in a neighbourhood of $\gamma_{0}$ where $B_{n}=O_{P}(1)$ and $\sup _{\theta \in \Theta}\left|Q_{n}\left(\theta, \gamma_{0}\right)-Q\left(\theta, \gamma_{0}\right)\right| \stackrel{P}{\rightarrow}$ 0 ; (iv) $\theta \mapsto Q\left(\theta, \gamma_{0}\right)$ is continuous and has a unique maximum at $\theta_{0}$. Then $A .1(i)$ and A.3 hold.

As a first step in our higher-order analysis, we prove in Appendix B (Lemma 7) that under Assumptions A.1-A.3,

$$
\hat{\theta}_{n, S}-\hat{\theta}_{n}=-H_{0}^{-1}\left\{G_{n}\left(\theta_{0}, \hat{\gamma}_{S}\right)-G_{n}\left(\theta_{0}, \gamma_{0}\right)\right\}+o_{P}(1 / \sqrt{n})
$$

We then evaluate a functional Taylor expansion of the leading right-hand side term w.r.t. $\hat{\gamma}_{S}$ around $\gamma_{0}$ :

A.4 $(m)$ There exist functionals $\nabla^{k} g(z, \theta, \gamma)\left[d \gamma_{1}, \ldots, d \gamma_{k}\right]$ for $(\theta, \gamma)$ in a neighbourhood of $\left(\theta_{0}, \gamma_{0}\right)$, and constants $\delta>0$ and $\bar{G}_{k}>0, k=0, \ldots, m$, such that: (i) each $\nabla^{k} g$ is linear in each of its components $d \gamma_{i} \in \Gamma, i=1, \ldots, k$; (ii)

$$
E\left[\left\|g\left(z, \theta, \gamma_{0}+d \gamma\right)-g\left(z, \theta, \gamma_{0}\right)-\sum_{k=1}^{m} \frac{1}{k !} \nabla^{k} g(z, \theta, \gamma)[d \gamma, \ldots, d \gamma]\right\|\right] \leq \bar{G}_{0}\|d \gamma\|^{m+1}
$$

\footnotetext{
${ }^{2}$ These cases could be handled by introducing a smoothed version of the approximators; see McFadden (1989), Fermanian and Salanié (2004), or Bruyns et al (2015). Alternatively, one could resort to empirical process theory, as done in Armstrong et al (2015).
} 
where $E\left[\|\nabla g(z, \theta, \gamma)[d \gamma]\|^{2}\right] \leq \bar{G}_{1}\|d \gamma\|^{2}$ and, for some $\nu>0$ and for $k=2, \ldots, m$, $E\left[\left\|\nabla^{k} g(z, \theta, \gamma)\left[d \gamma_{1}, \ldots, d \gamma_{k}\right]\right\|^{2+\nu}\right] \leq \bar{G}_{k}\left(\left\|d \gamma_{1}\right\| \cdots\left\|d \gamma_{k}\right\|\right)^{2+\nu}$

Assumption A.4 $(m)$ restricts $g(z, \theta, \gamma)$ to be $m$ times pathwise differentiable w.r.t. $\gamma$ with differentials $\nabla^{k} g(z)\left[d \gamma_{1}, \ldots, d \gamma_{k}\right]$ that are Lipschitz in $d \gamma_{1}, \ldots, d \gamma_{k}, k=1, \ldots, m$. For a given choice of $m$, this allows us to use an $m$ th order expansion of $G_{n}(\theta, \gamma)$ w.r.t. $\gamma$ to evaluate the impact of $\hat{\gamma}_{S}$. In particular, the difference between the approximate and the exact objective functions can be written as

$$
G_{n}\left(\theta_{0}, \hat{\gamma}_{S}\right)-G_{n}\left(\theta_{0}, \gamma_{0}\right)=\sum_{k=1}^{m} \frac{1}{k !} \nabla^{k} G_{n}\left(\theta_{0}, \gamma_{0}\right)\left[\hat{\gamma}_{S}-\gamma_{0}, \ldots, \hat{\gamma}_{S}-\gamma_{0}\right]+R_{n, S}
$$

where $R_{n, S}=O_{P}\left(\left\|\hat{\gamma}_{S}-\gamma_{0}\right\|^{m+1}\right)$ is the remainder term, and $\nabla^{k} G_{n}(\theta, \gamma)\left[d \gamma_{1}, \ldots, d \gamma_{k}\right]=$ $\sum_{i=1}^{n} \nabla^{k} g\left(z_{i}, \theta, \gamma\right)\left[d \gamma_{1}, \ldots, d \gamma_{k}\right] / n$. To evaluate the higher-order errors due to the approximation, we will study the mean and variance of each of the terms in the sum on the right hand side of (6).

\subsection{Approximators}

To analyze the impact of approximations, we need to further specify how the approximator behaves. Let us first introduce two alternative ways of implementing the approximation: Either one common approximator is used across all observations, or a new approximator is used for each observation. To differentiate between the two approximation schemes, we will refer to the approximate estimator based on the first scheme as an estimator based on common approximators (ECA) and to the second one as an estimator based on individual approximators (EIA):

$$
\mathbf{E C A}: G_{n}\left(\theta, \hat{\gamma}_{S}\right)=\frac{1}{n} \sum_{i=1}^{n} g\left(z_{i} ; \theta, \hat{\gamma}_{S}\right), \quad \mathbf{E I A}: G_{n}\left(\theta, \hat{\gamma}_{S}\right)=\frac{1}{n} \sum_{i=1}^{n} g\left(z_{i} ; \theta, \hat{\gamma}_{i, S}\right)
$$

In the first case, a single approximator $\hat{\gamma}_{S}$ is used the computation of the moment conditions across observations, while in the second one $n$ approximators $\hat{\gamma}_{1, S}, \ldots . \hat{\gamma}_{n, S}$ are used in the computation. We stress that the ECA and EIA are both targeting the same infeasible estimator; the only difference lies in how the approximators are used in the computation of the objective function.

Earlier papers on simulation-based methods (e.g. Laroque and Salanié, 1989; McFadden, 1989) used EIAs, and most papers on cross-sectional or panel data still do. ECAs were proposed by Lee (1992) for cross-sectional discrete choice models, but have mostly been used for dynamic models (Duffie and Singleton, 1993; Altissimo and Mele, 2010). To provide a streamlined set of regularity conditions that apply to both of these approximation schemes, 
we let $J \geq 1$ denote the number of approximators used in the computation of $\hat{\theta}_{n, S}$. For ECAs and EIAs, $J=1$ and $J=n$, respectively.

Next, we impose regularity conditions on the bias component of the approximator (which is common amongst the $J$ approximators) and its stochastic component defined by:

$$
b_{S}(u ; \theta):=E\left[\hat{\gamma}_{i, S}(u ; \theta) \mid u\right]-\gamma_{0}(u ; \theta), \quad \psi_{i, S}(u ; \theta):=\hat{\gamma}_{i, S}(u ; \theta)-E\left[\hat{\gamma}_{i, S}(u ; \theta) \mid u\right],
$$

for $i=1, \ldots, J$.

A.5 $(p)$ The approximator(s) lies in $\Gamma$ and satisfies:

(i) The $J(=1$ or $=n)$ random functions $\hat{\gamma}_{1, S}(u ; \theta), \ldots ., \hat{\gamma}_{J, S}(u ; \theta)$ are identically distributed, mutually independent and independent of $\mathcal{Z}_{n}$.

(ii) Their common bias $b_{S}$ is of order $\beta>0, b_{S}(u ; \theta)=S^{-\beta} \bar{b}(u ; \theta)+o\left(S^{-\beta}\right)$.

(iii) For $2 \leq q \leq p$, the stochastic component satisfies $E\left[\left\|\psi_{i, S}(u ; \theta)\right\|^{q}\right]=S^{-\alpha_{q}} v_{q}(u ; \theta)+$ $o\left(S^{-\alpha_{q}}\right), i=1, \ldots, J$, for some constant $\alpha_{q}>0$.

A.5(iii) requires the approximator to have $p$ moments and that each of these vanish at a given rate as $S \rightarrow \infty$. We will choose $p$ in conjunction with the order of the expansion $m$ of Assumption A.4, since we wish to evaluate the mean and variance of each of the higher-order terms. For example, in order to ensure that the variance of $\nabla^{k} G_{n}\left[\hat{\gamma}_{S}, \ldots, \hat{\gamma}_{S}\right]$ exists and to evaluate its rate of convergence, we will require A.5 $(p)$ to hold with $p=2 k$.

If $\hat{\gamma}_{S}$ is non-stochastic, as with numerical integration (Lee, 2001), discretization (Tauchen, 1986), or numerical inversion of a function, $\psi_{i, S}(u ; \theta)=0$ so that $\alpha_{p}=+\infty$ for all $p \geq 2$, and only a bias component is present. Stochastic approximation schemes, on the other hand, can involve both a bias and variance component. Monte Carlo schemes are the most prominent and we therefore specialize some of our results to the following class of Monte Carlo approximators:

A.6 $(p)$ The approximator $\hat{\gamma}_{i, S}(u ; \theta)$ takes the form $\hat{\gamma}_{i, S}(u ; \theta)=\sum_{s=1}^{S} w_{S}\left(u, \varepsilon_{i, s} ; \theta\right) / S, i=$ $1, \ldots, J$, where: (i) $\left\{\varepsilon_{i, s}\right\}_{s=1}^{S}$ is stationary and geometrically $\beta$-mixing; (ii) $\left\{\varepsilon_{i, s}\right\}_{s=1}^{S}$ and $\left\{\varepsilon_{j, s}\right\}_{s=1}^{S}$ are independent for $i \neq j$, and they are all independent of the sample; (iii) the function $w_{S}\left(u, \varepsilon_{i, s} ; \theta\right)$ satisfies, with expectations being taken w.r.t. $\varepsilon_{i, s}$,

$$
\bar{w}_{S}(u ; \theta):=E\left[w_{S}\left(u, \varepsilon_{i, s} ; \theta\right) \mid u\right]=\gamma_{0}(u ; \theta)+S^{-\beta} \bar{b}(u ; \theta)+o\left(S^{-\beta}\right)
$$

and for every $2 \leq q \leq p$, there exists $\mu_{q}<q / 2$ such that $E\left[\left\|w_{S}\left(u, \varepsilon_{i, s} ; \theta\right)-\bar{w}_{S}(u ; \theta)\right\|^{q} \mid u\right]=$ $O\left(S^{\mu_{q}}\right)$.

To our knowledge, A.6 includes all simulation-based approximators proposed in the literature, including Markov Chain Monte Carlo methods. The assumption of $\beta$-mixing is only 
used in the proof of Theorem 6, and could be weakened to "strongly mixing" elsewhere. Bias and variance rates of approximators satisfying A.6 follow from the assumptions imposed on $w_{S}$ : Using Jensen's inequality, $E\left[\|X\|^{q}\right] \leq E\left[\|X\|^{p}\right]^{q / p}$ for $q \leq p$, we see that $\mu_{q} \leq q \mu_{p} / p$ for $2 \leq q \leq p$. Given this inequality, it is easily verified that Assumption A.6 implies A.5 with the same rate $\beta$ for the bias term and with $\alpha_{q}=p / 2-\mu_{q}>0$ in A.5(iii).

In parametric simulation-based estimation, the approximator has no bias: $b_{S} \equiv 0$ and so $\beta=\infty$. Moreover, Assumption A.6(iii) typically holds with $\mu_{p}=0$, and A.5(iii) with $\alpha_{p}=$ $p / 2$. Methods where a bias component is present include nonparametric SMLE (NPSMLE) (Fermanian and Salanié, 2004; Kristensen and Shin, 2012), nonparametric SMM (Creel and Kristensen, 2012), and sieve approximated value functions (Kristensen and Scherning, 2012; Norets, 2009, 2012).

\section{Effects of Approximations}

We are now ready to derive the leading bias and variance terms of the estimator due to approximation errors. In the following, when we discuss biases and variances, we refer to the means and variances of the leading terms of a valid stochastic expansion of the estimators. This is a standard approach in the higher-order analysis of estimators; see, for example, Rothenberg (1984) and Newey and Smith (2004, section 3).

Let $g_{i}:=g\left(z_{i}, \theta_{0}, \gamma_{0}\right) ; \nabla g_{i}[d \gamma]:=\nabla g\left(z_{i}, \theta_{0}, \gamma_{0}\right)[d \gamma]$ and $\nabla^{2} g_{i}[d \gamma, d \gamma]:=\nabla^{2} g\left(z_{i}, \theta_{0}, \gamma_{0}\right)[d \gamma, d \gamma]$ for any function $d \gamma$. The leading terms in the bias of the approximate estimator then take the form

$$
B_{S, 1}=-H_{0}^{-1} E\left[\nabla g_{i}\left[b_{S}\right]\right] \text { and } B_{S, 2}=-\frac{1}{2} H_{0}^{-1} E\left[\nabla^{2} g_{i}\left[\psi_{i, S}, \psi_{i, S}\right]\right]
$$

where $b_{S}$ and $\psi_{i, S}$ are defined in eq. (8). The first bias term $B_{S, 1}$ is zero for unbiased approximators, as in parametric simulation-based inference. The second one, $B_{S, 2}$, is zero for non-stochastic approximators of the type found in numerical approximation schemes. The leading variance term due to the presence of approximations is $\nabla G_{n}\left(\theta_{0}\right)\left[\hat{\gamma}_{S}-\gamma\right]$. It can be decomposed into two terms. The first one is $D_{n, S}=\sum_{i=1}^{n} d_{i, S} / n$, where $d_{i, S}=$ $\nabla g_{i}\left[b_{S}\right]-E \nabla g_{i}\left[b_{S}\right]$, which is common to the two approximation schemes. The asymptotic properties of the second variance component, $E_{n, S}=\sum_{i=1}^{n} \nabla g_{i}\left[\psi_{i, S}\right] / n$ depend on whether we use EIA or ECA, however. The variance components $\psi_{i, S}$ vary across observations for EIAs; as a consequence, one can directly apply a CLT for stationary and mixing sequences to $E_{n, S}$. On the other hand, ECAs only have one $\psi_{S}$, which is common across observations, and getting a CLT takes more work and additional assumptions. We start by rewriting $E_{n, S}$ 


$$
E_{n, S}=\frac{1}{n} \sum_{i=1}^{n}\left\{\nabla g_{i}\left[\psi_{S}\right]-\nabla G\left[\psi_{S}\right]\right\}+\nabla G\left[\psi_{S}\right], \quad \text { with } \nabla G\left[\psi_{S}\right]:=E\left[\nabla g_{i}\left[\psi_{S}\right] \mid \psi_{S}\right]
$$

The first term is $O_{P}\left(S^{-\alpha_{2} / 2} / \sqrt{n}\right)$, and so is dominated by the second term $\nabla G\left[\psi_{S}\right]=$ $O_{P}\left(S^{-\alpha_{2} / 2}\right)$. In general, the large-sample distribution of $\nabla G\left[\psi_{S}\right]$ is not known in closedform. However, if we strengthen Assumption A.5 to A.6, we can write

$$
\nabla G\left[\psi_{S}\right]=\frac{1}{S} \sum_{s=1}^{S} \nabla G\left[e_{s, S}\right], \text { with } e_{s, S}\left(\varepsilon_{s}\right):=w_{S}\left(u, \varepsilon_{s} ; \theta_{0}\right)-E\left[w_{S}\left(u, \varepsilon_{s} ; \theta_{0}\right)\right]
$$

and a CLT can be applied as $S \rightarrow \infty$. The above terms make up the first-order expansion of the effects of approximations on the estimators:

Theorem 2 Assume A.1-A.3, A.4(2), and A.5(4). Then:

$\hat{\theta}_{n, S}-\theta_{0}=B_{S, 1}+B_{S, 2}+H_{0}^{-1}\left\{G_{n}+D_{n, S}+E_{n, S}\right\}+O_{P}\left(S^{-3 \beta}\right)+O_{P}\left(S^{-\alpha_{3}}\right)+o_{P}(1 / \sqrt{n})$,

where $G_{n}:=G_{n}\left(\theta_{0}, \gamma_{0}\right)$ and the two sequences $\left(G_{n}, D_{n, S}\right)$ and $E_{n, S}$ are asymptotically mutually independent. Moreover, the following limit results hold as $n, S \rightarrow \infty$ :

- For both EIA and ECA approximators,

$$
\sqrt{n}\left(\Omega_{S}^{G+D}\right)^{-1 / 2}\left\{G_{n}+D_{n, S}\right\} \stackrel{d}{\rightarrow} N\left(0, I_{k}\right), \text { with } \Omega_{S}^{G+D}=\lim _{n \rightarrow \infty} \frac{1}{n} \operatorname{Var}\left(\sum_{i=1}^{n} g_{i}+d_{i, S}\right)
$$

and $\Omega_{S}^{G+D}=\Omega^{G}+O\left(S^{-2 \beta}\right)$ with $\Omega^{G}=\frac{1}{n} \operatorname{Var}\left(\sum_{i=1}^{n} g_{i}\right)$.

- The bias terms have orders $B_{S, 1}=O\left(S^{-\beta}\right)$ and $B_{S, 2}=O\left(S^{-\alpha_{2}}\right)$.

- For EIA approximators, $\operatorname{Var}\left(E_{n, S}\right)=O_{P}\left(S^{-\alpha_{2}} n^{-1}\right)$; for ECA approximators, $\operatorname{Var}\left(E_{n, S}\right)=$ $O_{P}\left(S^{-\alpha_{2}}\right)$.

A first application of the theorem is to provide rates on the degree of approximation under which the approximate estimator is asymptotically first-order equivalent to the exact estimator; that is, which choices of the sequence $S=S_{n}$ guarantee $\left\|\hat{\theta}_{n, S_{n}}-\hat{\theta}_{n}\right\|=o_{P}\left(n^{-1 / 2}\right)$ ? In general, asymptotic equivalence for ECAs obtain if $n / S^{\min \left(\alpha_{2}, 2 \beta\right)} \rightarrow 0$; for EIA's we have a weaker condition, replacing $\alpha_{2}$ with $2 \alpha_{2}$. For parametric simulation-based estimators $(\beta=\infty$ and $\alpha_{2}=1$ ), this gives the standard result that $n / S_{n}$ should go to zero for ECA's (Duffie and Singleton, 1993; Lee, 1995, Theorem 1), while $\sqrt{n} / S_{n}$ should go to zero for EIA's (Laroque 
and Salanié, 1989; Lee, 1995, Theorem 4). Section 6.1 takes up the more complicated case of nonparametric kernel methods, as used in NPSML.

For the family of approximators satisfying Assumption 6, we can obtain a more precise characterization of the variance term $E_{n, S}$ :

Corollary 3 Assume that A.1-A.3, A.4(2), and A.6(4) hold, and $w=w_{S}$ does not depend on $S$. Then $\alpha_{2}=1$ and

$$
\begin{aligned}
& \text { EIA }: \sqrt{n S} E_{n, S} \stackrel{d}{\rightarrow} N\left(0, \Omega_{E I A}^{E}\right), \text { with } \Omega_{E I A}^{E}=\lim _{S \rightarrow \infty} \frac{1}{S} \operatorname{Var}\left(\sum_{s=1}^{S} \nabla g_{0}\left[e_{s}\right]\right), \\
& \text { ECA }: \sqrt{S} E_{n, S} \stackrel{d}{\rightarrow} N\left(0, \Omega_{E C A}^{E}\right), \text { with } \Omega_{E C A}^{E}=\lim _{S \rightarrow \infty} \frac{1}{S} \operatorname{Var}\left(\sum_{s=1}^{S} \nabla G\left[\tilde{e}_{s}\right]\right),
\end{aligned}
$$

where $e_{s}(u)=e_{s, S}(u)=w\left(u, \varepsilon_{s} ; \theta_{0}\right)-E\left[w\left(u, \varepsilon_{s} ; \theta_{0}\right)\right]$ is defined in eq. (10).

This corollary allow us to analyze the effects due to approximation errors in more detail. In particular, both EIA's and ECA's are normally distributed as $n, S \rightarrow \infty$ with leading bias and variance terms due to approximations given by:

$$
E\left[\hat{\theta}_{n, S}-\theta_{0}\right] \simeq B_{S, 1}+B_{S, 2}, \quad \operatorname{Var}\left(\hat{\theta}_{n, S}-\theta_{0}\right) \simeq H_{0}^{-1}\left\{\Omega_{S}^{G+D} / n+\operatorname{Var}\left(E_{n, S}\right)\right\} H_{0}^{-1}
$$

The bias and the variance of the approximator enter the two leading bias terms of the approximate estimator separately: the bias $b_{S}$ drives $B_{S, 1}$, and the stochastic components $\psi_{j, S}$ drive $B_{S, 2}$. When the approximator is a simple unbiased simulated average, $B_{S, 1}=0$ and the leading bias term $B_{S, 2}=O(1 / S)$; this is a well-known result for specific simulationbased estimators in cross-sectional settings - see e.g. Gouriéroux and Monfort (1996) and Lee (1995). Our theorem shows that this result holds more generally under weak regularity conditions.

EIA's and ECA's differ regarding the second variance term $E_{n, S}$. In the computation of the ECA, one common approximator is used across all observations; this introduces additional correlations across observations. In contrast, for EIA, $\psi_{i, S}$ and $\psi_{j, S}$ are independent for $i \neq j$. As a consequence, the variance due to a given number $S$ of simulations is larger for ECA's; and in leading simulation-based inference cases with $\beta=\infty$ and $\alpha_{2}=1$, we need $S$ to go to infinity faster than $n$ to keep the variance from exploding. This seems to suggest that one should prefer EIA to ECA; but statistical efficiency must be traded off with computational efficiency. If for instance $\hat{\gamma}_{S}$ is costly to implement, it may be convenient to use the same approximator across all observations.

The sharpness of the rates in Theorem 2 depends on the type of approximator being used and how it enters into the objective function; that is, the precise nature of the mapping 
$\gamma \mapsto g(z, \theta, \gamma)$

Theorem 4 Under the assumptions of Theorem 2, if the rates in Assumption A.5 are sharp then: (i) For non-stochastic approximators, all rates listed in the Theorem are sharp. (ii) For EIA's with $\nabla^{2} g_{i}[d \gamma, d \gamma] \neq 0$, the rates of $B_{S, 1}$ and $B_{S, 2}$ and $D_{n, S}$ and $E_{n, S}$ are sharp. If additionally Assumption A.6(4) holds with $w_{S} \equiv w$, the same is true for ECA's.

The proof of Theorem 4 follows from the arguments in the proof of Theorem 2 together with rate results for sample averages. Note that it does not cover nonparametric simulators, for which $w_{S}$ depends on $S$ through the bandwidth. If for instance $\hat{\gamma}_{S}$ is a kernel estimator and ECA is used, one can show that $\operatorname{Var}\left(E_{n, S}\right)=O\left(S^{-1}\right)$. Since $\alpha_{2}<1$ in this case, this bound is sharper than the rate stated in the theorem; see Creel and Kristensen (2012) and Kristensen and Shin (2012).

In some special cases, a term in the expansion is zero. In SMM for instance, the function $g$ is linear in the approximator $\gamma$. Then $\nabla^{2} g_{i}[d \gamma, d \gamma]=0$, so that $B_{S, 2}=0$; and our rates are obviously not sharp. On the other hand, for nonparametric approximation methods, such as NPSML, all of the terms may be simultaneously nonzero if $\gamma$ enters non-linearly. This follows directly from the coexistence of bias and variance in nonparametric smoothers; see Section 6.1 .

\section{Newton-Raphson and Jackknife Adjustment}

We here propose two methods that remove some of the additional biases and variances in estimation due to approximations. The first is a Newton-Raphson type adjustment that reduces both bias and variance of the approximate estimator, while the second aims at removing biases only. Hajivassiliou (2000, section 3) proposed using Newton-Raphson for SML, but it has not been used much. Bruyns et al (2015, section 4) also recommend both Newton-Raphson and jackknifing.

The Newton-Raphson adjustment works for both stochastic and non-stochastic approximations. Our proposal builds on the well-known result that a consistent estimator can be made asymptotically efficient by applying one Newton-Raphson (NR) step of the loglikelihood function to it. E.g. if $\hat{\theta}_{n}$ is a $\sqrt{n}$-consistent estimator of $\theta_{0}$, then a single NR-step yields a consistent and asymptotically efficient estimator. We extend this idea to our setting by starting from some initial approximate estimator based on a degree of approximation $S$, say $\bar{\theta}_{n, S}$. We then define the corrected estimator through one or possibly several NewtonRaphson iterations of an approximate objective function that uses a much finer approximation, $S^{*} \gg S$. With $H_{n}(\theta, \gamma)=\partial G_{n}(\theta, \gamma) /(\partial \theta)$, we define iteratively

$$
\hat{\theta}_{n, S}^{(k+1)}=\hat{\theta}_{n, S}^{(k)}-H_{n}^{-1}\left(\hat{\theta}_{n, S}^{(k)}, \hat{\gamma}_{S^{*}}\right) G_{n}\left(\hat{\theta}_{n, S}^{(k)}, \hat{\gamma}_{S^{*}}\right), \quad k=1,2,3, \ldots
$$


where $\hat{\theta}_{n, S}^{(1)}=\bar{\theta}_{n, S}$, and we use the $S^{*}$ th order approximator, $\hat{\gamma}_{S^{*}}$, in the iterations. It should be noted that instead of the inverse of the exact Hessian, $H_{n}^{-1}(\theta, \gamma)$, one could employ an estimate of this, say, $W_{n}(\theta, \gamma)$, in the above Newton-Raphson adjustment. This could, for example, be due to the use of numerical (instead of analytical) derivatives or, as in the socalled BHHH algorithm, the use of the cross-product of the vector of first derivatives in place of the second-order derivatives. This however will slow down the convergence rate and the result of Theorem 5 below has to be adjusted, c.f. Robinson (1988, Theorem 5). In particular, more iterations are required to obtain a given level of precision.

If $G_{n}(\theta, \gamma)=\partial Q_{n}(\theta, \gamma) /(\partial \theta)$, then the cost of computing each new iterate from the previous one is (very) roughly $S^{*} / S$ times the cost of one iteration in the minimization of $Q_{n}\left(\theta, \hat{\gamma}_{S^{*}}\right)$. Since the minimization itself can easily require a hundred iterations or so, we can therefore take $S^{*}$ ten or twenty times larger than $S$ without adding much to the cost of the estimation procedure. If $G_{n}(\theta, \gamma)$ is a set of moment conditions, the above Newton-Raphson method can be modified to avoid having to compute second-order derivatives. Using the notation of Example 2, the modified version of the above Newton-Raphson algorithm takes the form

$$
\hat{\theta}_{n, S}^{(k+1)}=\hat{\theta}_{n, S}^{(k)}+\left(\hat{H}_{n} W_{n} \hat{H}_{n}\right)^{-1} \hat{H}_{n} W_{n} M_{n}\left(\hat{\theta}_{n, S}^{(k)}, \hat{\gamma}_{S^{*}}\right),
$$

where $\hat{H}_{n}$ is a consistent estimator of $H\left(\theta_{0}, \gamma_{0}\right)=\partial M\left(\theta_{0}, \gamma_{0}\right) /(\partial \theta)$, c.f. Newey and McFadden (1994, p. 2150-2151).

To evaluate the performance of $\hat{\theta}_{n, S}^{(k+1)}$ relative to $\bar{\theta}_{n, S^{*}}$, we first note that $\left\|\hat{\theta}_{n, S}^{(k+1)}-\hat{\theta}_{n}\right\| \leq$ $\left\|\hat{\theta}_{n, S}^{(k+1)}-\bar{\theta}_{n, S^{*}}\right\|+\left\|\bar{\theta}_{n, S^{*}}-\hat{\theta}_{n}\right\|$. Combining this with Robinson (1988, Theorem 2), we obtain the following theorem:

Theorem 5 Assume that A.1-A.3, A.4(3) and A.5(6) hold. Let the initial estimate $\bar{\theta}_{n, S}$ be consistent. Then the NR-estimator $\hat{\theta}_{n, S}^{(k+1)}$ defined in (13) satisfies $\left\|\hat{\theta}_{n, S}^{(k+1)}-\hat{\theta}_{n}\right\|=O_{P}\left(\| \bar{\theta}_{n, S}-\right.$ $\left.\hat{\theta}_{n} \|^{2^{k}}\right)+O_{P}\left(\left\|\bar{\theta}_{n, S^{*}}-\hat{\theta}_{n}\right\|\right)$ as $n, S$ and $S^{*}$ go to infinity with $S^{*}>S$.

This result formalizes the intuition that a large enough number of NR-steps with the score and Hessian evaluated at $\gamma_{S^{*}}$ yields an estimator that is equivalent to the extremum estimator obtained from full optimization of the objective function based on $\gamma_{S^{*}}$. This holds irrespective of the convergence rate of the initial estimator. However, the number of NR iterations, $k$, needed to obtain this result does depend on the precision of the initial estimator. For unadjusted parametric simulation-based estimators in the EIA scheme for instance, we know from Theorem 2 that $\left\|\bar{\theta}_{n, S}-\hat{\theta}_{n}\right\|=O_{P}(1 / S)$. Then the first term on the right-hand side of the inequality in Theorem 5 is asymptotically dominated by the second term if $S^{*}=o\left(S^{2^{k}}\right)$. Taking $k=1$ and having $S^{*} / S$ converge to some positive number would be enough in this case.

Jackknifing could be used as an alternative or a complement to Newton-Raphson iter- 
ations. Recall from Theorem 2 that $E\left[\hat{\theta}_{n, S}-\hat{\theta}_{n}\right] \simeq b_{1} S^{-\beta}+b_{2} S^{-\alpha_{2}}$. First compute two approximators of order $S^{*}$ which we denote $\hat{\gamma}_{S^{*}}^{[1]}$ and $\hat{\gamma}_{S^{*}}^{[2]}$. Let $\hat{\theta}_{n, S^{*}}^{[m]}$ be the estimator based on the same data sample $\mathcal{Z}_{n}$ but using the $m$ th approximator $\hat{\gamma}_{S^{*}}^{[m]}, m=1,2$. Then consider the following jackknife (JK) type estimator:

$$
\hat{\theta}_{n, S}^{\mathrm{JK}}:=2 \hat{\theta}_{n, S}-\frac{1}{2}\left\{\hat{\theta}_{n, S^{*}}^{[1]}+\hat{\theta}_{n, S^{*}}^{[2]}\right\} .
$$

It is easy to see that

$$
\begin{aligned}
E\left[\hat{\theta}_{n, S}^{\mathrm{JK}}-\hat{\theta}_{n}\right] & =2 E\left[\hat{\theta}_{n, S}-\hat{\theta}_{n}\right]-\frac{1}{2}\left\{E\left[\hat{\theta}_{n, S^{*}}^{[1]}-\hat{\theta}_{n}\right]+E\left[\hat{\theta}_{n, S^{*}}^{[2]}-\hat{\theta}_{n}\right]\right\} \\
& \simeq b_{1}\left\{2 S^{-\beta}-\left(S^{*}\right)^{-\beta}\right\}+b_{2}\left\{2 S^{-\alpha}-\left(S^{*}\right)^{-\alpha_{2}}\right\}
\end{aligned}
$$

where we ignored higher-order terms. We would now ideally choose $S^{*}$ such that both of the above bias terms cancel out. However, we can only remove either of the two: By choosing either $S^{*}=S / 2^{1 / \beta}$ or $S^{*}=S / 2^{1 / \alpha_{2}}$, we will remove the first or the second term respectively. Obviously, $S^{*}$ should be chosen so as to remove the bias component that dominates in the expansion. In a previous version we also reported results for this resampling method; and we tested it on the mixed logit model that we explore in section 7 . We found that the improvements from resampling were dominated by those obtained with the other methods.

\section{Analytical Adjustments}

The expansions derived in section 3 naturally suggest correcting the approximate estimators and standard errors to take into account the biases and variances due to approximations. The corrections are obtained by constructing consistent estimators of the leading terms in the formulæ of Theorem 2, and Corollary 3 when applicable.

\subsection{Bias Adjustment}

The leading bias terms are $B_{S, 1}$ and $B_{S, 2}$. We mainly focus on the case where $\beta>\alpha_{2}$. Recall that this includes parametric simulation-based estimation methods, but it excludes most purely non-stochastic approximators. Then $B_{S, 1}$ is of lower order and the leading bias component is $B_{S, 2}=-\frac{1}{2} H_{0}^{-1} \nabla^{2} G_{S}$, where $\nabla^{2} G_{S}:=E\left[\nabla^{2} g\left(z_{i} ; \theta_{0}, \gamma_{0}\right)\left[\psi_{i, S}, \psi_{i, S}\right]\right]$.

We wish to adjust the approximate estimator to remove this bias component. The two main approaches to bias adjustment in the econometric literature are "corrective" and "preventive". The corrective method first computes the unadjusted estimator, $\hat{\theta}_{n, S}$, obtains a consistent estimator of the bias, $\hat{B}_{S, 2}$, and then combines the two to obtain a new, biasadjusted (BA) estimator, $\tilde{\theta}_{n, S}^{\mathrm{BA}}=\hat{\theta}_{n, S}-\hat{B}_{S, 2}$. One example of this approach can be found in Lee (1995) for the special case of SMLE and SNLS in limited dependent variable models. 
A natural estimator of $\hat{B}_{S, 2}$ would be $\hat{B}_{S, 2}=-\frac{1}{2} \hat{H}_{n}^{-1} \nabla^{2} \hat{G}_{n, S}$ for some consistent estimator $\nabla^{2} \hat{G}_{n, S}$ of $\nabla^{2} G_{S}$. We propose two different estimators depending on whether A.6 holds or not. If A.6 does not apply, the following estimator is available for EIA:

$$
\text { EIA }: \nabla^{2} \hat{G}_{n, S}=\frac{1}{n} \sum_{i=1}^{n} \nabla^{2} g\left(z_{i} ; \hat{\theta}_{n, S}, \hat{\gamma}_{S}\right)\left[\hat{\psi}_{i, S}, \hat{\psi}_{i, S}\right], \quad \hat{\psi}_{i, S}:=\hat{\gamma}_{i, S}-\frac{1}{n} \sum_{i=1}^{n} \hat{\gamma}_{i, S} \text {. }
$$

For the ECA version, one cannot estimate the variance component of $\hat{\gamma}_{S}$ without further simulations. One possibility would be to simulate $m$ extra, mutually independent versions, $\hat{\gamma}_{k, S}$, $k=1, \ldots, m$, of $\hat{\gamma}_{S}$, and then compute $\nabla^{2} \hat{G}_{n, S}=\frac{1}{n m} \sum_{i=1}^{n} \sum_{k=1}^{m} \nabla^{2} g\left(z_{i} ; \hat{\theta}_{n, S}, \hat{\gamma}_{S}\right)\left[\hat{\psi}_{k, S}, \hat{\psi}_{k, S}\right]$, where $\hat{\psi}_{k, S}=\hat{\gamma}_{k, S}-\frac{1}{m} \sum_{k=1}^{m} \hat{\gamma}_{k, S}$. Here, $m$ has to be chosen large enough so that the variance component of $\nabla^{2} \hat{G}_{n}(\theta)$ does not dominate the bias that we are trying to remove. This means that the computational cost of this first ECA bias estimator can be large, expecially if $\hat{\gamma}_{S}$ is not easy to compute.

When A.6 also holds, the following alternative estimator is available; and it can be used for both ECA's and EIA's:

$$
\nabla^{2} \hat{G}_{n, S}=\frac{1}{n S(S-1)} \sum_{i=1}^{n} \sum_{s=1}^{S} \nabla^{2} g\left(z_{i} ; \hat{\theta}_{n, S}, \hat{\gamma}_{i, S}\right)\left[\hat{e}_{i, s, S}, \hat{e}_{i, s, S}\right]
$$

here, in the case of EIA's, $\hat{e}_{i, s, S}(u ; \theta)=w_{S}\left(u, \varepsilon_{i, s} ; \theta\right)-\hat{\gamma}_{i, S}(u ; \theta)$ while in the case of ECA, $\hat{e}_{i, s, S}(u ; \theta)=w_{S}\left(u, \varepsilon_{s} ; \theta\right)-\hat{\gamma}_{S}(u ; \theta)$ and so does not change across observations $i=1, \ldots, n$.

Instead of adjusting the estimator, we can do preventive correction where we adjust the estimating equation $G_{n}\left(\theta, \hat{\gamma}_{S}\right)$ to remove the component leading to the bias $B_{S, 2}$. By inspection of the proof of Theorem 2 , it is easily seen that the relevant adjustment of $G_{n}\left(\theta_{0}, \hat{\gamma}_{S}\right)$ is $\nabla^{2} G_{S} / 2$. This suggests a bias-adjusted estimator $\hat{\theta}_{n, S}^{\mathrm{BA}}$ that solves

$$
G_{n}\left(\hat{\theta}_{n, S}^{\mathrm{BA}}, \hat{\gamma}_{S}\right)-\frac{1}{2} \nabla^{2} \hat{G}_{n, S}\left(\hat{\theta}_{n, S}^{\mathrm{BA}}\right)=o_{P}(1 / \sqrt{n})
$$

where $\nabla^{2} \hat{G}_{n, S}(\theta)$ is taken either from eq. (16) or (under A6) from eq. (17), with $\hat{\theta}_{n, S}$ replaced by $\theta$. This approach was pursued in the context of SNLS (see Example 1) by Laffont et al (1995).

After either preventive or corrective adjustment, the bias component $B_{S, 2}$ is replaced by

$$
\tilde{B}_{S, 2}:=-\frac{1}{2} H_{0}^{-1}\left(\nabla^{2} G_{S}-E\left[\nabla^{2} \hat{G}_{n, S}\right]\right)
$$

The following theorem analyzes the properties of the bias adjusted estimator based on $\nabla^{2} \hat{G}_{n, S}$ given in eq. (17). We expect similar results to hold for any bias adjusted EIA estimator that uses eq. (16). 
Theorem 6 Assume that A.1-A.3, A.4(3), and A.6(8) hold together with

$$
\left\|\nabla^{2} g\left(z ; \theta_{0}\right)\left[e_{i s}, e_{i t}\right]\right\| \leq b(z)\left\|e_{i s}(z)\right\|\left\|e_{i t}(z)\right\|
$$

where $E\left[b^{8}(z)\right]<\infty$. Then any $\hat{\theta}_{n, S}^{\mathrm{BA}}$ solving eq. (18) satisfies as $n, S \rightarrow \infty$ :

$$
\begin{aligned}
\hat{\theta}_{n, S}^{\mathrm{BA}}-\theta_{0}= & B_{S, 1}+\tilde{B}_{S, 2}+H_{0}^{-1}\left\{G_{n}+D_{n, S}+E_{n, S}\right\} \\
& +O_{P}\left(S^{-3 \beta}\right)+O_{P}\left(S^{-2+\mu_{4}}\right)+O\left(S^{-2+\mu_{3}}\right)+o_{P}(1 / \sqrt{n}),
\end{aligned}
$$

where $\tilde{B}_{S, 2}$ given in eq. (19) satisfies $\tilde{B}_{S, 2}=O\left(S^{-2+\mu_{2}}\right)$ and $\mu_{p}, p \geq 2$, is defined in A.6. All other terms in the expansion are as in Theorem 2.

Note that under the assumptions of Theorem $6,-2+\mu_{4}<0,-2+\mu_{3}<-1 / 2$ and $-2+\mu_{2}<-1$. The theorem therefore shows that under slightly stronger conditions ${ }^{3}$ than in Theorem 2, $\tilde{B}_{S, 2}$ has a faster rate of convergence than $B_{S, 2}$, while the rate of the other leading terms is unchanged. More precisely, compared to Theorem 2, the bias term $B_{S, 2}=$ $O\left(S^{-\alpha_{2}}\right)=O\left(S^{-1+\mu_{2}}\right)$ has been replaced by $\tilde{B}_{S, 2}=O\left(S^{-2+\mu_{2}}\right)$. Also note that the higherorder variance component of order $O_{P}\left(S^{-\alpha_{3}}\right)$ that appeared in Theorem 2 has been replaced by $O_{P}\left(S^{-2+\mu_{4}}\right)+O\left(S^{-2+\mu_{3}}\right)$. In the proof, we show that the variance of $\nabla^{2} \hat{G}_{S}$, that we use to estimate $B_{S, 2}$, is of order $O_{P}\left(n^{-1 / 2} S^{-1+\mu_{8} / 4}\right)+O_{P}\left(n^{-1 / 2} S^{-1+\alpha_{4} / 2}\right)=o_{P}(1 / \sqrt{n})$. In particular, the additional variances that we introduce when estimating the bias are of smaller order than the bias being adjusted for and so the bias adjusted estimator dominates the unadjusted one.

With unbiased simulators, we have $\mu_{2}=0$ and $\beta=\infty$, and by Theorem 2 the leading bias term of the unadjusted estimator is of order $O\left(S^{-1}\right)$. Theorem 6 shows that for the adjusted estimator the leading term of the bias is of order $O\left(S^{-2}\right)$. The improvement is by a factor $S$ and may be quite large. More generally, the proposed adjustment will remove the largest bias component as long as $\alpha_{2}<\beta$. Otherwise the bias term $O_{P}\left(S^{-\beta}\right)$ is of a larger order than $O_{P}\left(S^{-\alpha_{2}}\right)$ and the proposed bias adjustment does not remove the leading term anymore. In particular, when non-stochastic approximations are employed the above adjustment does not help. If we could estimate $b_{S}$, then $B_{S, 1}$ could be taken care of easily by adjusting either estimator or estimating equation using $\nabla \hat{G}_{n, S}:=\sum_{i=1}^{n} \nabla g_{i}\left(\hat{\theta}_{n, S}, \hat{\gamma}_{S}\right)\left[\hat{b}_{S}\right] / n$. However, estimating $b_{S}$ can be a difficult task.

\subsection{Adjusting Standard Errors}

If the approximator is stochastic, the approximate estimator will not only be biased; it will also contain additional variance terms, c.f. eq. (12). We should adjust inferential tools (such

\footnotetext{
${ }^{3}$ The higher order on A.6 is required to ensure that in the asymptotic expansion, the remainder term $R_{n, S}$ is still dominated.
} 
as standard errors and $t$-statistics) to account for these additional variances. This turns out to be quite straightforward in many cases. To keep the notation simple, we assume in the following that data and simulations are i.i.d. ${ }^{4}$.

The different terms appearing in the variance expansion in eq. (12) implicitly depend on $\theta_{0}$ and $\gamma_{0}$. In standard estimation procedures, one would usually estimate the above variance components by simply replacing $\theta_{0}$ and $\gamma_{0}$ by $\hat{\theta}_{n, S}$ and $\hat{\gamma}_{S}$, respectively, in the expressions of $\Omega^{G+D}, \operatorname{Var}\left(E_{n, S}\right)$ and $H_{0}$, and by replacing any population means by their sample counterparts. The variance term $\Omega_{S}^{G+D}$ involves the bias component of the approximator, $b_{S}$. This is unknown in most cases, but we know from Theorem 2 that $\Omega_{S}^{G+D}=\Omega^{G}+O\left(S^{-2 \beta}\right)$ where $\Omega^{G}=E\left[g\left(z, \gamma_{0}\right) g\left(z, \gamma_{0}\right)^{\prime}\right]$. For large $S$, a simple estimator would therefore be

$$
\hat{\Omega}_{S}^{G+D}=\hat{\Omega}^{G}=\frac{1}{n} \sum_{i=1}^{n} \hat{g}_{i} \hat{g}_{i}^{\prime} \text {, where } \hat{g}_{i}=g\left(z_{i} ; \hat{\theta}_{n, S}, \hat{\gamma}_{S}\right)
$$

However, replacing $\gamma_{0}$ by $\hat{\gamma}_{S}$ will generate biases. Similarly, if $\hat{\theta}_{n, S}$ has not been bias adjusted, replacing $\theta_{0}$ by $\hat{\theta}_{n, S}$ will add biases to the variance estimator. Specifically, under suitable regularity conditions and by the same arguments as employed in the proof of Theorem 2 ,

$$
E\left[\hat{\Omega}^{G}\right]=\Omega^{G}+O\left(S^{-\beta}\right)+O\left(S^{-\alpha_{2}}\right) .
$$

Recall that either $\operatorname{Var}\left(E_{n, S}\right)=O_{P}\left(S^{-\alpha_{2}} n^{-1}\right)$ (EIA) or $\operatorname{Var}\left(E_{n, S}\right)=O_{P}\left(S^{-\alpha_{2}}\right)$ (ECA), and so the biases in eq. (20) will often be of the same order as the variance components that we are trying to adjust for.

We therefore propose a bias-adjusted estimator of $\hat{\Omega}^{G}$ to improve on the basic variance estimators in the same way that we bias-adjusted $G_{n}\left(\theta, \hat{\gamma}_{S}\right)$. We assume in the following that $\hat{\theta}_{n, S}$ has already been bias adjusted so that we only need to adjust any biases due to $\hat{\gamma}_{S}$. This adjustment takes the form $\tilde{\Omega}_{\mathrm{BA}}^{G}=\hat{\Omega}^{G}-\hat{\Delta}_{n, S}^{\Omega}$ where either, in the case of EIA's with $\hat{\psi}_{i, S}:=\hat{\gamma}_{i, S}-\bar{\gamma}_{S}$,

$$
\text { EIA : } \hat{\Delta}_{n, S}^{\Omega}=\frac{1}{n} \sum_{i=1}^{n}\left\{\nabla^{2} \hat{g}_{i}\left[\hat{\psi}_{i, S}, \hat{\psi}_{i, S}\right] \hat{g}_{i}^{\prime}+2 \nabla \hat{g}_{i}\left[\hat{\psi}_{i, S}\right] \nabla \hat{g}_{i}\left[\hat{\psi}_{i, S}\right]^{\prime}+\hat{g}_{i} \nabla^{2} \hat{g}_{i}\left[\hat{\psi}_{i, S}, \hat{\psi}_{i, S}\right]^{\prime}\right\}
$$

or, under Assumption A.6 for both EIA and ECA,

$\hat{\Delta}_{n, S}^{\Omega}=\frac{1}{n S(S-1)} \sum_{i=1}^{n} \sum_{s=1}^{S}\left\{\nabla^{2} \hat{g}_{i}\left[\hat{e}_{i, s, S}, \hat{e}_{i, s, S}\right] \hat{g}_{i}^{\prime}+2 \nabla \hat{g}_{i}\left[\hat{e}_{i, s, S}\right] \nabla \hat{g}_{i}\left[\hat{e}_{i, s, S}\right]^{\prime}+\hat{g}_{i} \nabla^{2} \hat{g}_{i}\left[\hat{e}_{i, s, S}, \hat{e}_{i, s, S}\right]^{\prime}\right\} ;$

here $\hat{e}_{i, s, S}$ is defined as right after eq. (17). The analysis of this estimator proceeds as in the proof of Theorem 6 .

\footnotetext{
${ }^{4}$ Otherwise long-run variance estimators have to be used.
} 
Next consider $\operatorname{Var}\left(E_{n, S}\right)$. As we know from Theorem 2, the behaviour of this term depends on whether EIA or ECA are used. In the case of EIA, $\operatorname{Var}\left(E_{n, S}\right) \simeq \operatorname{Var}\left(\nabla g_{i}\left[\psi_{i, S}\right]\right) / n$ which can be estimated by $\widehat{\operatorname{Var}}\left(E_{n, S}\right)=\sum_{i=1}^{n} \nabla g_{i}\left[\psi_{i, S}\right] \nabla g_{i}\left[\psi_{i, S}\right]^{\prime} / n^{2}$.

When ECA is employed, $\operatorname{Var}\left(E_{n, S}\right) \simeq \operatorname{Var}\left(\nabla G\left[\psi_{S}\right]\right)$ which can be estimated by $\widehat{\operatorname{Var}}\left(E_{n, S}\right)=$ $\sum_{k=1}^{m} \nabla \hat{G}\left[\psi_{S, k}\right] \nabla \hat{G}\left[\psi_{S, k}\right]^{\prime} / m$, where $\psi_{S, k}=\hat{\gamma}_{S, k}-\sum_{k=1}^{m} \hat{\gamma}_{S, k} / m, \hat{\gamma}_{S, k}, k=1, \ldots, m$, are $m \geq 1$ independent versions of $\hat{\gamma}_{S}$ distribution of $\psi_{S}$, and $\nabla \hat{G}[d \gamma]=\sum_{i=1}^{n} \nabla \hat{g}_{i}[d \gamma] / n$. This can be time consuming if $\hat{\gamma}_{S}$ is a costly to compute.

The proposed estimators will suffer from biases similar to the ones in $\hat{\Omega}^{G}$, but these biases are of smaller order compared to the variance adjustment that we are making.

If Assumption A.6 holds, better estimates can be obtained since in this case Corollary 3 yields either $\operatorname{Var}\left(E_{n, S}\right) \simeq \Omega_{\mathrm{EIA}}^{E} /(n S)(\mathrm{EIA})$ or $\operatorname{Var}\left(E_{n, S}\right) \simeq \Omega_{\mathrm{ECA}}^{E} / S$ (ECA) where $\Omega_{\mathrm{EIA}}^{E}=$ $\operatorname{Var}\left(\nabla g_{i}\left[\bar{w}_{i, s}\right]\right)$ and $\Omega_{\mathrm{ECA}}^{E}=\operatorname{Var}\left(\nabla G\left[\bar{w}_{s}\right]\right)$, and we have assumed for simplicity that the simulations are independent across $s=1, \ldots, S$. This suggests the following simple estimators,

$$
\hat{\Omega}_{\mathrm{EIA}}^{E}=\frac{1}{n S} \sum_{i=1}^{n} \sum_{s=1}^{S} \nabla \hat{g}_{i}\left[\hat{e}_{i, s, S}\right] \nabla \hat{g}_{i}\left[\hat{e}_{i, s, S}\right]^{\prime} \text { and } \hat{\Omega}_{\mathrm{ECA}}^{E}=\frac{1}{S} \sum_{s=1}^{S} \nabla \hat{G}\left[e_{s, S}\right] \nabla \hat{G}\left[\hat{e}_{s, S}\right]^{\prime},
$$

where $\nabla \hat{G}[\gamma]=\sum_{i=1}^{n} \nabla g\left(z_{i}, \hat{\theta}_{n, S}, \hat{\gamma}_{S}\right)[\gamma] / n$. The estimator $\hat{\Omega}_{\text {ECA }}^{E}$ is similar to the one proposed in Newey (1991) for semiparametric two-step estimators.

For EIA, two terms cancel out when we combine $\hat{\Delta}_{n, S}^{\Omega}$ with $\hat{\Omega}_{\mathrm{EIA}}^{E}$, giving

$$
\hat{\Delta}_{n, S}^{\Omega}-\hat{\Omega}_{\mathrm{EIA}}^{E}=\frac{1}{n S^{2}} \sum_{i=1}^{n} \sum_{s=1}^{S}\left\{\nabla^{2} \hat{g}_{i}\left[\hat{e}_{i, S}, \hat{e}_{i, S}\right] \hat{g}_{i}^{\prime}+\nabla \hat{g}_{i}\left[\hat{e}_{i, S}\right] \nabla \hat{g}_{i}\left[\hat{e}_{i, S}\right]^{\prime}+\hat{g}_{i} \nabla^{2} \hat{g}_{i}\left[\hat{e}_{i, S}, \hat{e}_{i, S}\right]^{\prime}\right\}
$$

Finally, the naive estimator of $H_{0}$ takes the form $\hat{H}=H_{n}\left(\hat{\theta}_{n, S}, \hat{\gamma}_{S}\right)$. One could bias-adjust this estimator as we did for $\hat{\Omega}^{G}$. However, note that the approximate estimator satisfies $o_{P}(1 / \sqrt{n})=G_{n}\left(\theta_{0}, \hat{\gamma}_{S}\right)+H_{n}\left(\bar{\theta}_{n, S}, \hat{\gamma}_{S}\right)\left(\hat{\theta}_{n, S}-\theta_{0}\right)$, for some $\bar{\theta}_{n, S}$ on the line segment connecting $\hat{\theta}_{n, S}$ and $\theta_{0}$. So in order to get a precise approximation of the distribution of $\hat{\theta}_{n, S}-\theta_{0}$, we want to use an estimator that mimics the behaviour of $H_{n}\left(\bar{\theta}_{n, S}, \hat{\gamma}_{S}\right)$. This is exactly what $\hat{H}$ does.

To sum up, for EIA, we propose the following bias-adjusted variance estimator for $\hat{\theta}_{n, S}$,

$$
\hat{H}^{-1} \frac{1}{n} \sum_{i=1}^{n}\left(\hat{g}_{i} \hat{g}_{i}^{\prime}-\frac{1}{S^{2}} \sum_{s=1}^{S}\left(\nabla^{2} \hat{g}_{i}\left[\hat{e}_{i, S}, \hat{e}_{i, S}\right] \hat{g}_{i}^{\prime}+\nabla \hat{g}_{i}\left[\hat{e}_{i, S}\right] \nabla \hat{g}_{i}\left[\hat{e}_{i, S}\right]^{\prime}+\hat{g}_{i} \nabla^{2} \hat{g}_{i}\left[\hat{e}_{i, S}, \hat{e}_{i, S}\right]^{\prime}\right)\right) \hat{H}^{-1}
$$

while for ECA it takes the form $\hat{H}^{-1}\left(\hat{\Omega}^{G}-\hat{\Delta}_{n, S}^{\Omega}+\hat{\Omega}_{\mathrm{ECA}}^{E}\right) \hat{H}^{-1}$. 


\section{Applications}

We here show the applicability of our general results, by analyzing two particular approximate estimators.

\subsection{Simulated maximum likelihood}

For SML we approximate the density $p(z ; \theta)\left(=\gamma_{0}\right)$ so that $g(z ; \theta, p)=p_{\theta}(z ; \theta) / p(z ; \theta)$, where $p_{\theta}(z ; \theta)=\partial p(z ; \theta) /(\partial \theta)$, is the score of the log-likelihood. Then, suppressing dependence on $(z ; \theta)$,

$$
\nabla g[d p]=\frac{d p_{\theta}}{p}-\frac{p_{\theta}}{p^{2}} d p \quad \text { and } \quad \nabla^{2} g[d p, d p]=\frac{2 p_{\theta}}{p^{3}}(d p)^{2}-\frac{2}{p^{2}} d p d p_{\theta},
$$

so that $\bar{G}_{0}$ in A.4 involves higher-order moments of $1 / p$. If the density $p\left(z ; \theta_{0}\right) \rightarrow 0$ as $\|z\| \rightarrow$ $\infty$, these moments may not be finite. One can introduce trimming, replacing the simple simulator $\hat{p}_{S}(z ; \theta)$ described above with $\hat{p}_{a, S}(z ; \theta)=\hat{p}_{S}(z ; \theta) \tau_{a}\left(\hat{p}_{S}(z ; \theta)\right)$ where $\tau_{a}(w)$ is a smooth trimming function that satisfies $\tau_{a}(w)=1$ for $w \geq 2 a$ and $\tau_{a}(w)=0$ for $w \leq a$. Then $\bar{G}_{a, 0}=O\left(a^{-(m+1)}\right)$ is finite for any $a>0$, and the remainder term satisfies $R_{n, S}=$ $O_{P}\left(a^{-(m+1)}\left\|\hat{p}_{a, S}-p\right\|^{m+1}\right)$. By letting $a=a_{S} \rightarrow 0$ at a suitable rate as $S \rightarrow \infty$, it is now possible to control the remainder term while the expansion remains valid; see Creel and Kristensen (2012) and Kristensen and Shin (2012) for more details in the context of SMM and SMLE, respectively.

The analytical adjustments are easy to compute when the approximator $\hat{p}_{S}$ satisfies A.6 with $\beta=\infty$. Assume for instance that it uses independent simulations (the EIA case.) Denoting $r_{i, s}=w_{S}\left(z_{i}, \varepsilon_{i, s} ; \theta\right)-\hat{p}_{i}, \hat{p}_{i}=\hat{p}_{S}\left(z_{i} ; \theta\right), \hat{p}_{\theta, i}=\partial \hat{p}_{S}\left(z_{i} ; \theta\right) /(\partial \theta)$, and so forth, we obtain the following expression for the bias adjustment,

$$
\nabla^{2} \hat{G}_{S}(\theta)=\frac{2}{n S} \sum_{i=1}^{n}\left(\frac{\hat{p}_{\theta, i}}{\hat{p}_{i}^{3}} \frac{1}{S} \sum_{s=1}^{S} r_{i, s}^{2}-\frac{1}{\hat{p}_{i}^{2}} \frac{1}{S} \sum_{s=1}^{S} r_{i, s} \dot{r}_{i, s}\right) .
$$

Our proposed analytical adjustment to the variance of the estimators replaces the standard variance estimator, $n^{-1} \sum_{i=1}^{n} p_{\theta, i} p_{\theta, i}^{\prime} / p_{i}^{2}$, by

$$
\frac{1}{n} \sum_{i=1}^{n}\left(\frac{\hat{p}_{\theta, i} \hat{p}_{\theta, i}^{\prime}}{\hat{p}_{i}^{2}}-\frac{1}{S^{2}} \sum_{s=1}^{S}\left(\frac{r_{\theta, i, s}^{2}}{\hat{p}_{i}^{2}}+9 \frac{r_{i, s}^{2} \hat{p}_{\theta, i} \hat{p}_{\theta, i}^{\prime}}{\hat{p}_{i}^{4}}-4 \frac{r_{i, s}\left(r_{\theta, i, s}^{\prime} \hat{p}_{\theta, i}^{\prime}+\hat{p}_{\theta, i}^{\prime} r_{\theta, i, s}^{\prime}\right)}{\hat{p}_{i}^{3}}\right)\right) .
$$

It is sometimes not possible to obtain an unbiased simulator of a density; then the NPSML estimator offers an attractive alternative. Suppose that the model takes the form $y=m\left(x, \varepsilon, \theta_{0}\right)$ and we compute $y_{s}(x, \theta)=m\left(x, \varepsilon_{s}, \theta_{0}\right), s=1, \ldots, S$. The nonparametric simulated density then satisfies A.6 with $w_{S}\left(y, x, \varepsilon_{s} ; \theta\right)=K_{h}\left(y_{s}(x, \theta)-y\right)$ where the band- 
width $h=h(S) \rightarrow 0$ as $S \rightarrow \infty$. Let $d=\operatorname{dim}(y)$ and suppose that we use a kernel of order $r$. The bias component satisfies $\bar{w}_{S}(y, x ; \theta)-p(y \mid x ; \theta)=h^{r} \frac{\partial^{r} p(y \mid x ; \theta)}{\partial y^{r}}+o\left(h^{r}\right)$. Furthermore, it is easily checked that $E\left[\left|K_{h}\left(y_{s}(x, \theta)-x\right)\right|^{p} \mid x\right]=O\left(-h^{d(p-1)}\right)$ for all $p \geq 2$ under suitable regularity conditions. Thus, with a bandwidth of order $h \propto S^{-\delta}$ for some $\delta>0$, A.6(p) holds with $\beta=r \delta$ and $\mu_{p}=\delta d(p-1)$ for $p \geq 2$. We only need to choose $\delta<p /(2 d(p-1))$ so that $\mu_{p}<p / 2$.

As is well-known, the asymptotic mean integrated squared error is smallest when the bias and variance component are balanced. This occurs when $\delta^{*}=1 /(2 r+d)$, leading to $\beta=r /(2 r+d)$. It is easy to check that these values satisfy A6(p) if $r>d(p-2) /(2 p)$, which allows for the standard choice of $r=2$ except in implausibly high-dimensional cases. We recover of course the standard nonparametric rate. ${ }^{5}$

Let us now return to first-order efficiency. Using standard arguments from the literature on semiparametric estimation, one can show in great generality that $\Omega_{\mathrm{ECA}}^{E}=O\left(S^{-1}\right)$ (see Kristensen and Shin, 2012 for further details). Given this result, it easily follows from Theorem 2 that for the NPSMLE based on ECA's to be equivalent to the MLE, we need $\sqrt{n} h^{r} \rightarrow 0, n / S \rightarrow 0$ and $\sqrt{n} /\left(S h^{d}\right)^{2} \rightarrow 0$. For EIA's, $\Omega_{\mathrm{EIA}}^{E}=O\left(1 /\left(n S h^{d+2}\right)\right)$ and so $n / S \rightarrow 0$ has to be replaced by $\sqrt{n} S h^{d+2} \rightarrow \infty$.

We derive in Appendix C.1 the analytical adjustments for such an NPSML estimator when $y$ is scalar $(d=1)$ and the data is i.i.d. Given some additional regularity conditions, we obtain

$$
\begin{aligned}
& B_{S, 1} \simeq-h^{r} \frac{\kappa_{r}}{r !} H_{0}^{-1} E\left[b_{1, i}\left(\theta_{0}\right)\right], \\
& B_{S, 2} \simeq \frac{1}{S h^{d}} \int K^{2}(z) d z \times H_{0}^{-1} E\left[\frac{p_{\theta, i}\left(\theta_{0}\right)}{p_{i}^{2}\left(\theta_{0}\right)}\right]-\frac{1}{S h^{d+1}} \int K(z) K^{\prime}(z) d z \times H_{0}^{-1} E\left[\frac{m_{\theta, i}\left(\theta_{0}\right)}{p_{i}\left(\theta_{0}\right)}\right],
\end{aligned}
$$

with $\kappa_{r}=\int K(z) z^{r} d z, H_{0}=E\left[p_{\theta, i}\left(\theta_{0}\right) p_{\theta, i}\left(\theta_{0}\right)^{\prime} / p_{i}^{2}\left(\theta_{0}\right)\right]$,

$$
b_{1, i}(\theta):=\frac{p_{\theta, i}(\theta)}{p_{i}^{2}(\theta)} \frac{\partial^{r} p_{i}(\theta)}{\partial y_{i}^{r}}-\frac{1}{p_{i}(\theta)} \frac{\partial^{r} p_{\theta, i}(\theta)}{\partial y_{i}^{r}}
$$

and $m_{\theta, i}(\theta)=\partial m\left(x_{i}, r\left(x_{i}, y_{i}\right) ; \theta\right) /(\partial \theta)$. Here, we use " $\simeq$ " to indicate that only leading terms are included. From these expressions, we see that the kernel smoother distorts the NPSMLE by an order of magnitude $O\left(h^{r}\right)$ while the simulations, in conjunction with the smoothing, generate additional biases of order $O\left(1 /\left(S h^{d+1}\right)\right)$ and $O\left(1 /\left(S h^{d}\right)\right)$. If a symmetric kernel is employed, $\int K(z) K^{\prime}(z) d z=0$ and the second term in the expression of $B_{S, 2}$ drops out. Standard bandwidth selection rules in general imply $S h^{d} \rightarrow \infty$, but this

\footnotetext{
${ }^{5}$ While the standard nonparametric rate is optimal for the approximation of the individual densities that make up the likelihood, this rate does not yield the best NPSML estimators. This is akin to results for semiparametric two-step estimators where undersmoothing of the first-step nonparametric estimator is normally required for the parametric estimator to be $\sqrt{n}$-consistent; see Kristensen-Salanié (2010) for details.
} 
is it not enough for the bias to vanish with rate $\sqrt{n}$; we need to undersmooth so that $\sqrt{n} /\left(S h^{d+1}\right) \rightarrow 0$.

The variance components satisfy $D_{n, S} \simeq-\frac{\kappa_{r}}{r !}\left(h^{r} / n\right) \sum_{i=1}^{n}\left\{b_{1, i}\left(\theta_{0}\right)-E\left[b_{1, i}\left(\theta_{0}\right)\right]\right\}$ and $\operatorname{Var}\left(E_{n, S}\right) \simeq\left(n S h^{d+2}\right)^{-1} E\left[\sigma_{\theta, i}^{2}\left(\theta_{0}\right) / p_{i}\left(\theta_{0}\right)\right] \int K^{\prime}(z)^{2} d z$, where $\sigma_{\theta, i}^{2}(\theta)=\operatorname{Var}\left(m_{\theta, i}(\theta) \mid x_{i}\right)$. Note that when EIA is employed, the rate of the correction to the variance is non-standard compared to standard SML, which has an efficiency loss of order $1 / S$.

\subsection{Newton-Raphson on SMM estimators}

To illustrate the use of the Newton-Raphson algorithm in the case of GMM estimators, as given in eq. (14), we here provide the necessary formulæ for the case of the standard empirical IO model of Berry, Levinsohn and Pakes (1995). In this model, agent $k$ on market $i$ chooses between $J$ alternatives (products) based on utilities

$$
u_{k j i}=x_{j i}\left(\beta+A \varepsilon_{k i}\right)+\xi_{j i}+v_{k j i}, \quad j=1, \ldots, J
$$

where $v_{k j i}$ are iid standard type-I EV errors, $x_{j i} \in \mathbb{R}^{p}, \beta \in \mathbb{R}^{p}, A=\left[a_{q r}\right]_{q, r}$ is a $(p \times d)$ scaling matrix, and $\varepsilon_{k i}$ are iid with known multivariate $\operatorname{cdf} F$ and pdf $f$. We collect the unknown parameters in $\theta=(\beta, A)$. Let $\gamma_{0, j}(x, \xi ; \theta) \in[0,1]$ be market share function of market $j$ defined as $\gamma_{0, j}(x, \xi ; \theta)=\int \nu_{j}(\theta, x, \xi, \varepsilon) f(\varepsilon) d \varepsilon$ where

$$
\nu_{j}(x, \xi, \varepsilon ; \theta)=\frac{\exp \left(x_{j}^{\prime}(\beta+A \varepsilon)+\xi_{j}\right)}{1+\sum_{k=1}^{J} \exp \left(x_{k}(\beta+A \varepsilon)+\xi_{k}\right)}, \quad j=1, \ldots, J .
$$

We observe covariates $x_{i}$, market shares $m_{i} \in \mathbb{R}^{J}$, and a set of instruments $w_{i}, i=1, \ldots, n$, satisfying

$$
m_{i}=\gamma_{0}\left(x_{i}, \xi_{i} ; \theta_{0}\right) \text { and } E\left[w_{i} \otimes \xi_{i}\right]=0,
$$

where $\gamma_{0}=\left(\gamma_{0,1}, \ldots, \gamma_{0, J}\right)$ is the vector of market share functions, and then wish to estimate $\theta$ by GMM.

The market share functions cannot be written in analytical form, and the literature approximates them by

$$
\hat{\gamma}_{S, i}(x, \xi ; \theta)=\frac{1}{S} \sum_{s=1}^{S} \nu\left(x, \xi, \varepsilon_{i, s} ; \theta\right),
$$

where $\varepsilon_{i, 1}, \ldots, \varepsilon_{i, S}$ are i.i.d. draws from $F, i=1, \ldots, n$. We here employ the EIA scheme with independent draws across markets. Our simulated moment conditions are then given by

$$
M_{n}\left(\theta, \hat{\gamma}_{S}\right)=\frac{1}{n} \sum_{i=1}^{n} w_{i} \otimes \hat{\gamma}_{S, i}^{-1}\left(x_{i}, m_{i} ; \theta\right)
$$


where $\hat{\gamma}_{S, i}^{-1}(x, m ; \theta)$ is the inverse of the function $\hat{\gamma}_{S, i}(x, \xi ; \theta)$ w.r.t. $\xi$.

We show in Appendix C.2 that the matrix $\hat{H}_{n}=\partial M_{n}\left(\bar{\theta}_{n, S}, \hat{\gamma}_{S^{*}}\right) /(\partial \theta)$ in eq. (14) can be evaluated with the following simple and cost-effective procedure for a given estimator $\bar{\theta}_{n, S}$ :

1. compute the choice probabilities $\hat{\nu}_{i s} \equiv \nu\left(x_{i}, \hat{\xi}_{i}, \varepsilon_{s i} ; \bar{\theta}_{n, S}\right)$ for all markets $i=1, \ldots, n$ and $s=1, \ldots, S^{*}$, where $\hat{\xi}_{i}=\hat{\gamma}_{S^{*}, i}^{-1}\left(x_{i}, m_{i} ; \bar{\theta}_{n, S}\right)$

2. on each market $i$, compute the derivatives of $\hat{\gamma}_{i} \equiv \hat{\gamma}_{S^{*}, i}$ by

$$
\frac{\partial \hat{\gamma}_{S^{*}, i}}{\partial \xi}\left(x_{i}, \hat{\xi}_{i} ; \bar{\theta}_{n, S}\right)=\frac{1}{S^{*}} \sum_{s=1}^{S^{*}}\left(\operatorname{diag}\left(\hat{\nu}_{i s}\right)-\left(\hat{\nu}_{i s} \otimes \hat{\nu}_{i s}\right)\right),
$$

and for $j=1, \ldots, J$,

$$
\frac{\partial \hat{\gamma}_{S^{*}, i, j}}{\partial \beta}\left(x_{i}, \hat{\xi}_{i} ; \bar{\theta}_{n, S}\right)=\frac{1}{S^{*}} \sum_{s=1}^{S^{*}} \hat{\nu}_{j i s} \hat{x}_{j i s}, \quad \frac{\partial \hat{\gamma}_{S^{*}, i j}}{\partial A}\left(x_{i}, \hat{\xi}_{i} ; \bar{\theta}_{n, S}\right)=\frac{1}{S^{*}} \sum_{s=1}^{S^{*}} \hat{\nu}_{j i s}\left(\hat{x}_{j i s} \otimes \varepsilon_{s i}\right),
$$

with $\hat{x}_{j i s}$ the vector with components $x_{j i, q}-\sum_{l=1}^{J} \hat{\nu}_{l, s i} x_{l i, q}$ for $q=1, \ldots, p$.

3. Compute

$\frac{\partial \hat{\gamma}_{S^{*}, i}^{-1}}{\partial \theta}\left(x_{i}, m_{i} ; \bar{\theta}_{n, S}\right)=-\left[\frac{\partial \hat{\gamma}_{S^{*}, i}}{\partial \theta}\left(x_{i}, \hat{\gamma}_{S, i}^{-1}\left(x_{i}, m_{i} ; \bar{\theta}_{n, S}\right) ; \hat{\theta}_{n, S}\right)\right]^{-1} \frac{\partial \hat{\gamma}_{S^{*}, i}}{\partial \theta}\left(\hat{\gamma}_{S, i}^{-1}\left(x_{i}, m_{i} ; \bar{\theta}_{n, S}\right) ; \bar{\theta}_{n, S}\right)$

and substitute it into

$$
\hat{H}_{n}=\frac{1}{n} \sum_{t=1}^{n} w_{i} \otimes \frac{\partial \hat{\gamma}_{S^{*}, i}^{-1}}{\partial \theta}\left(x_{i}, m_{i} ; \bar{\theta}_{n, S}\right)
$$

\section{Simulation Study}

To explore the performance of our proposed approaches, we set up a small Monte Carlo study of the following mixed logit model (see Train, 2009, for more details and its applications): The econometrician observes i.i.d. draws of $z_{i}=\left(x_{i}, y_{i}\right)$ for $i=1, \ldots, n$, with $x_{i}$ a centered normal of variance $\tau^{2}$ and

$$
y_{i}=\mathbb{1}\left(b+\left(a+s u_{i}\right) x_{i}+e_{i}>0\right)
$$

where $e_{i}$ is standardized type I extreme value and $u_{i}$ is a centered normal with unit variance, independent of $e_{i}$.

We take the true model to have parameters $a=1, s=1, b=0$. In this specification, the mean probability of $y=1$ is one-half. For $\tau=1$ (resp. $\tau=2$ ) the generalized $R^{2}$ is 0.11 
(resp. 0.21); in the corresponding simple logit model, which has $s=0$, the $R^{2}$ would be 0.17 (resp. 0.39.)

We wish to estimate the model by MLE. Since the implied choice probabilities, given by

$$
\operatorname{Pr}(y=1 \mid x)=\int \frac{\phi(u)}{1+\exp (-(b+(a+s u) x))} d u \text {. }
$$

are not available on closed form, we implement the SMLE instead, c.f. Section 6.1

\subsection{Theoretical Fisher bounds and biases}

This is still a very simple model; thus we can use (adaptive) Gaussian quadrature to compute the conditional choice probabilities. Since Gaussian quadrature achieves almost correct numerical integration in such a regular, one-dimensional case, we can rely on it to do (almost) exact maximum likelihood estimation. By the same token, it is easy to compute the asymptotic variance of the exact ML estimator $\hat{\theta}_{n}$, and the leading term $B_{S, 2}$ of the bias of the SML estimator. Simple calculations give the numbers in Table 1.

\begin{tabular}{|r|r|r|r|r|r|r|}
\hline \hline$\tau$ & \multicolumn{3}{|c|}{$\sqrt{n} \hat{\sigma}$} & \multicolumn{3}{|c|}{$S$ times bias } \\
\cline { 2 - 7 } & $a$ & $s$ & $b$ & $a$ & $s$ & $b$ \\
\hline 1 & 7.2 & 17.1 & 2.4 & -9.0 & -23.2 & -0.0 \\
2 & 6.7 & 10.8 & 2.8 & -8.2 & -13.3 & -0.0 \\
\hline
\end{tabular}

Table 1: Rescaled asymptotic standard errors and simulation biases

The columns labeled $\sqrt{n} \hat{\sigma}$ give the square roots of the diagonal terms of the inverse of the Fisher information matrix. As can be seen from the values of $\sqrt{n} \hat{\sigma}$, it takes a large number of observations to estimate this model reliably. To take an example, assume that the econometrician would be happy with a modestly precise $95 \%$ confidence interval of halfdiameter 0.2 for the mean slope $a$. With $\tau=1$ it would take about $(7.2 * 1.96 / 0.2)^{2} \simeq 5,200$ observations; and still about 4,500 for $\tau=2$, even though the generalized $R^{2}$ almost doubles. With such sample sizes, the estimate of the size of the heterogeneity $s$ would still be very noisy: its $95 \%$ confidence intervals would have half-diameters 0.48 and 0.32 , respectively for $\tau=1$ and $\tau=2$. We also found that the correlation between the estimators of $a$ and of $s$ is always large and positive - of the order of 0.8. Thus the confidence region for the pair $(a, s)$ is in fact a rather elongated ellipsoid. On the other hand, the estimates of $b$ are reasonably precise, which is not very surprising as $b$ shifts the mean probability of $y=1$ strongly.

The figures in the columns labeled "S times bias" refer to the expansions of $\hat{\theta}_{n S}-\hat{\theta}_{n}$ in our theorems. We will be using SML under the EIA scheme (independent draws across observations). Then we know that the leading term of the bias due to the simulations is $B_{S, 2}$ and is of order $1 / S$. The figures give our numerical evaluation of $S B_{S, 2}$, using our 
formulæ and Gaussian quadrature again. As appears clearly from Table 1, once again the heterogeneity coefficient $s$ is the harder to estimate, followed by $a$, while there is hardly any bias on $b$. With $S=100$ simulations and $\tau=1$ for instance, the bias on $a$ is -0.09 , and the bias on $s$ is -0.23 .

\subsection{Experiments}

We ran experiments for several sets of parameter values, sample sizes $n$, explanatory power (through $\tau$ ), and numbers of draws $S$. Since the results are similar, we only present here those we obtained for a sample of 10,000 observations when the true model has $a=1, s=1, b=0$, and the covariate has standard error $\tau=1$ or $\tau=2$.

We present below the results for $S=50,100,200$, and 500 simulations. We ran 5,000 simulations in each case, starting from initial values of the parameters drawn randomly from uniform distributions: $a \sim U[0.5,1.5], b \sim U[-0.5,0.5]$, and $s \sim U[0.5,1.5]$. For each simulated sample with $S \leq 200$, we estimated the model using (i) uncorrected SML, (ii) SML with Newton-Raphson (NR), and (iii) SML with analytic adjustment (AA) for both bias and variance. The AA was done on the objective function. For the NR correction, we use only $k=1$ step, with $S^{*}=10 \times S$ draws $^{6}$.

For each method, we also used several ways of computing the standard errors of the estimates: from the most commonly used, which consists of inverting the outer product of the scores without correcting for the simulations, to the better-grounded sandwidch formula which we introduced in Section 6.

In order to maximize the simulated log-likelihood, we used the $C^{++}$version of the Minuit optimizer of $\mathrm{Cern}^{7}$, with the BFGS algorithm. We evaluated all gradients numerically, with one step of the Ridders-Richardson extrapolation method $^{8}$. We proceeded in the same way with the Hessians for the standard errors. We faced very few numerical difficulties. The optimization algorithm sometimes stopped very close to the bounds we had imposed for the heterogeneity parameter, $0.1 \leq s \leq 5$. In some cases it failed to find an optimum, especially for uncorrected SML with 50 draws. Finally, the second derivative of the simulated loglikelihood was sometimes not invertible in one of our sandwich formulæ. Altogether, we had to discard $0.2 \%$ to $3 \%$ of the 5,000 samples, depending on the run. When a sample fails, it is most often because the uncorrected SML does not converge, or it is hard to evaluate the corresponding standard errors. The corrected SML method appears to be much more robust. The tables and graphs below only refer to the remaining samples.

Two considerations are worth mentioning:

\footnotetext{
${ }^{6}$ We did not run the NR correction for $S=500$ as it would have been quite time-consuming, with little benefit.

${ }^{7}$ http://lcgapp.cern.ch/project/cls/work-packages/mathlibs/minuit/index.html.

${ }^{8}$ We experimented with up to four steps, but the gains in precision were negligible and the results were unchanged.
} 
- Ease of implementation: the analytical bias adjustment wins on that count, since it is usually easy to get a formula for the $\Delta$ term and to program it. The Newton method may be more troublesome in models with more than a few parameters, as it requires a reasonably accurate evaluation of the matrix of second derivatives. In our experiment, we relied on the fact that the minimization algorithm itself proceeds by Newton-Raphson steps; after multiplying by ten the number of simulations, we let the algorithm do exactly one iteration of its line search. This appears to work very well, and is very easy to implement.

- Computer time: it is important to compare methods that have similar run times. Table 2 reports the mean times per sample. The numbers in the table show that the analytical bias adjustment requires negligible computer resources. To evaluate the corrected objective function we only need to compute the variances of the simulated choice probabilities as well as their derivatives - a very small computational cost. Newton adjustment is clearly more demanding. The table shows that in our experiment, the Newton step itself was about two to three times as costly as uncorrected SML. For both values of $\tau$, "SML+Newton" with $S=50$ (and $S^{*}=500$ ) takes the same time as "SML" with $S=200$; and "SML+Newton" with $S=200$ (and $S^{*}=2,000$ ) takes about $20 \%$ longer than "SML" with $S=500$. We will use these two pairs in our evaluation of the Newton-Raphson method.

We should stress here that time comparisons can only be indicative, and here perhaps even more than usual since they depend on the structure of the model, on the difficulty of optimizing the log-likelihood, and on the care needed to approximate the Hessian. Note from the table that it is twice less expensive to $(a)$ use $S=50$ simulations to get an estimator and then take a Newton step with $S^{*}=500$ simulations than to $(b)$ work with $S^{*}=500$ simulations from the start. A simple calculation suggests that option $a$ should in fact be even more attractive in more complex models ${ }^{9}$. To see this, take any model whose computing cost mostly consists in evaluating the objective function. With $p$ parameters to estimate, maximizing the objective function takes a number of function calculations $F(p)$ that is (roughly) constant with the number of simulations; and the time requested to evaluate one function value is roughly proportional to the number of simulations $S$. This puts the cost of optimizing the objective function at $F(p) S$. Taking one Newton step has a cost $c(p) S^{*}$ if $c(p)$ function evaluations are needed. Unless the model has a large number of parameters (so that $c(p)$ may be large) and yet it is very easy to estimate (so that $F(p)$ is small), $c(p)$ is likely to be a small fraction of $F(p)$; and option $a$ will be cheaper than option $b$, as their respective computer costs are $\left(F(p) S+c(p) S^{*}\right)$ and $F(p) S^{*}$.

\footnotetext{
${ }^{9}$ We are grateful to a referee for prompting this discussion.
} 
Section 6.2 illustrates this: the formulæ for the Newton-Raphson adjustment can be written in closed form in empirical IO applications, making option $a$ much cheaper than reestimating the model with a larger number of simulations.

\begin{tabular}{|r|r||r|r|r|}
\hline \hline$\tau$ & \multicolumn{1}{|c||}{ S } & SML & SML+Newton & SML+AA \\
\hline \multirow{4}{*}{1} & 50 & 2.3 & 6.7 & 2.3 \\
& 100 & 3.8 & 11.9 & 3.9 \\
& 200 & 6.6 & 21.6 & 6.8 \\
& 500 & 17.3 & - & 17.7 \\
\hline \multirow{4}{*}{2} & 50 & 2.1 & 6.5 & 2.2 \\
& 100 & 3.6 & 11.7 & 3.8 \\
& 200 & 6.3 & 21.3 & 6.6 \\
& 500 & 16.7 & - & 17.2 \\
\hline
\end{tabular}

Table 2: Mean CPU time (seconds per sample)

\subsection{Results}

We focus on $a$ and $s$ as there is little to correct for in the SML estimates of $b$. We report (Huber) robust means, standard errors and RMSEs. "AA" refers to our analytical bias adjustment.

Tables 3 and 4 report our results for the mean error of our various SML methods. Each row corresponds to a value of the number of simulations $S$. All numbers in the last three columns of these tables were computed by averaging the "error terms" $\left(\hat{\theta}_{n, S}-\theta_{0}\right)$ over the 5,000 samples (minus the small number that were eliminated due to numerical issues.) The standard error of these averages is about 0.001 , so that several of the biases from the corrected estimates are statistically insignificant.

\begin{tabular}{|r|r||c|r|r|}
\hline \hline$\tau$ & \multicolumn{1}{|c||}{ S } & SML & SML+Newton & SML+AA \\
\hline & 50 & -0.133 & -0.089 & 0.004 \\
1 & 100 & -0.078 & -0.039 & 0.000 \\
& 200 & -0.041 & -0.014 & 0.000 \\
& 500 & -0.017 & - & 0.000 \\
\hline \multirow{4}{*}{2} & 50 & -0.133 & -0.051 & 0.010 \\
& 100 & -0.069 & -0.016 & -0.016 \\
& 200 & -0.033 & 0.003 & 0.006 \\
& 500 & -0.010 & - & 0.006 \\
\hline
\end{tabular}

Table 3: Mean error on $a$ 


\begin{tabular}{|r|r||c|r|r|}
\hline \hline$\tau$ & \multicolumn{1}{|c|}{ S } & SML & SML+Newton & SML+AA \\
\hline \multirow{4}{*}{1} & 50 & -0.364 & -0.217 & 0.011 \\
& 100 & -0.206 & -0.093 & 0.000 \\
& 200 & -0.109 & -0.033 & 0.001 \\
& 500 & -0.045 & - & 0.001 \\
\hline \multirow{4}{*}{2} & 50 & -0.214 & -0.064 & 0.021 \\
& 100 & -0.110 & -0.018 & -0.018 \\
& 200 & -0.051 & 0.010 & 0.013 \\
& 500 & -0.013 & - & 0.013 \\
\hline
\end{tabular}

Table 4: Mean error on $s$

The "SML" columns in the tables report the biases of the uncorrected SML estimator. The leading term appears to be a good approximation to the actual size of the bias in these simulations, and the measured bias is close to proportional to $1 / S$. This suggests that our analytical bias adjustment, which focuses on correcting for the leading term of the bias, should work very well. As the last columns show, AA in fact does eliminate most of the bias. The Newton step with ten times more simulations reduces the bias, as expected; but it does not do it as effectively as our analytical bias adjustment. In fact, comparing the SML estimator with $S=500$ to the Newtonized estimator $\left(S=50, S^{*}=500\right)$ shows that the Newton method only delivers part of the benefits suggested by the theory. Note that with $S=500$, there is not that much bias to correct, but what there is AA corrects quite well again.

The discussion above only bears on bias, but one may legitimately be concerned about the possibility that our adjustment procedures introduce more noise into the estimates and perhaps even increase their mean square errors. Tables 5 and 6 show that this concern is unfounded. Correcting the estimates using analytical adjustment or a Newton step reduces the RMSE in all cases. Most often, the reduction in bias dominates and AA works better than Newton. However, for larger number of simulations when $\tau=2$, bias reduction matters less; and since the Newton method is more effective at reducing dispersion, its RMSE becomes smaller than that of the AA method. This suggests that combining AA and a Newton step could yield an even larger reduction in the RMSE.

Both the bias and the increased variance imparted by the simulations affect the properties of standard tests. Figures 1 and 2 document this for $t$-tests that $a$ and $s$, respectively, equal their true values. For such a large sample, we would expect the distributions of the $t$-statistics to be very close to a standard centered normal; and $95 \%$ of the mass should lie between -1.96 and 1.96. What we observe for the uncorrected SML estimator ("SML") is quite different: the bias in the estimate skews the distribution to the left, spectacularly so for small number of simulations; and the increased variance flattens the distribution. 


\begin{tabular}{|r|r||r|r|r|}
\hline \hline$\tau$ & \multicolumn{1}{|c||}{ S } & SML & SML+Newton & SML+AA \\
\hline & 50 & 0.139 & 0.095 & 0.041 \\
1 & 100 & 0.083 & 0.043 & 0.028 \\
& 200 & 0.046 & 0.019 & 0.020 \\
& 500 & 0.021 & - & 0.013 \\
\hline & 50 & 0.136 & 0.053 & 0.032 \\
2 & 100 & 0.072 & 0.020 & 0.020 \\
& 200 & 0.036 & 0.010 & 0.016 \\
& 500 & 0.014 & - & 0.012 \\
\hline
\end{tabular}

Table 5: RMSE on $a$

\begin{tabular}{|r|r||r|r|r|}
\hline \hline$\tau$ & \multicolumn{1}{|c||}{ S } & SML & SML+Newton & SML+AA \\
\hline & 50 & 0.379 & 0.234 & 0.107 \\
1 & 100 & 0.219 & 0.103 & 0.074 \\
& 200 & 0.121 & 0.044 & 0.053 \\
& 500 & 0.056 & - & 0.033 \\
\hline \multirow{4}{*}{2} & 50 & 0.219 & 0.068 & 0.056 \\
& 100 & 0.115 & 0.025 & 0.025 \\
& 200 & 0.056 & 0.019 & 0.029 \\
& 500 & 0.021 & - & 0.022 \\
\hline
\end{tabular}

Table 6: RMSE on $s$ 


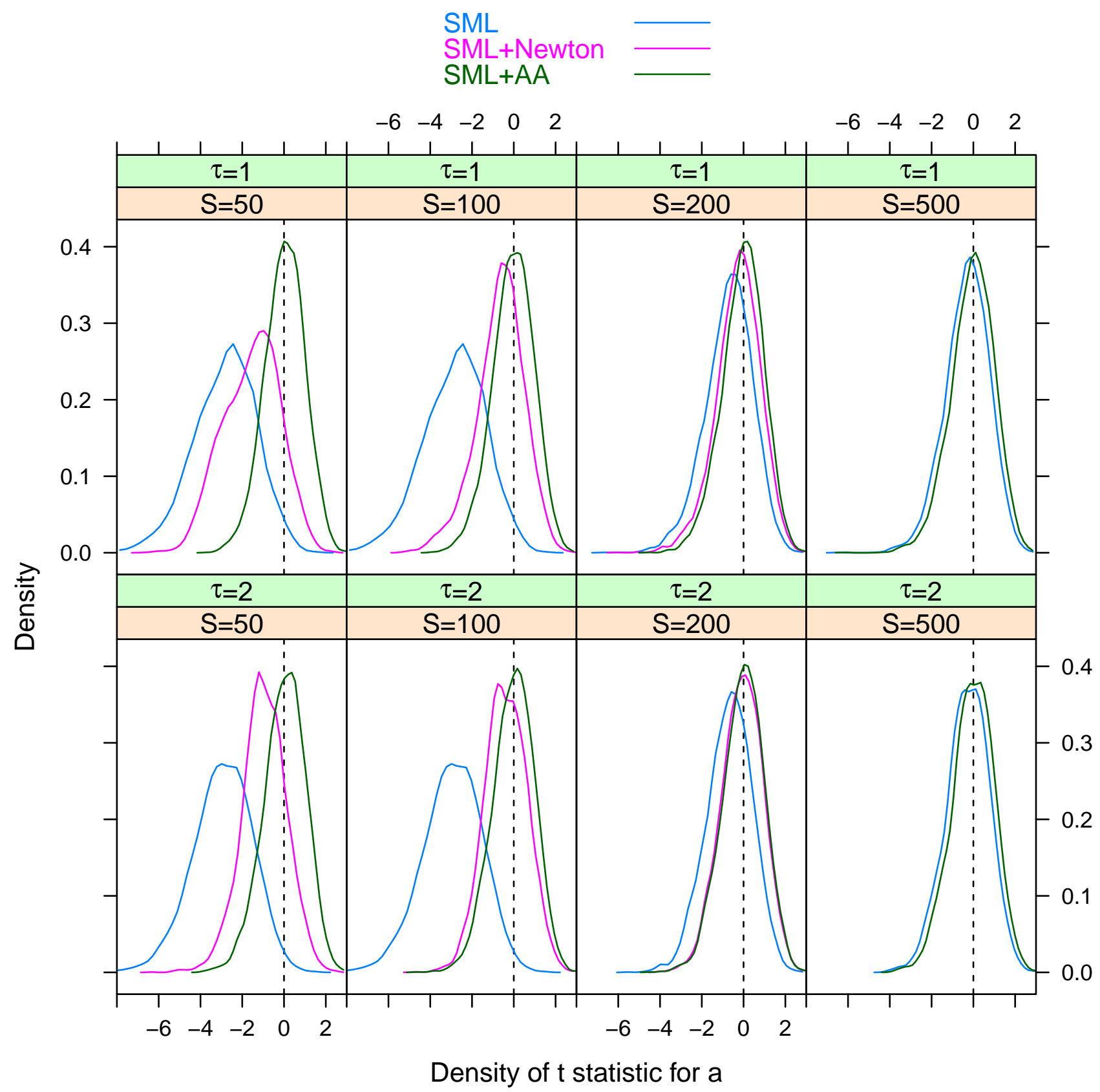

Figure 1: Distributions of the $t$ statistics for $\left(H_{0}\right): a=1$ 


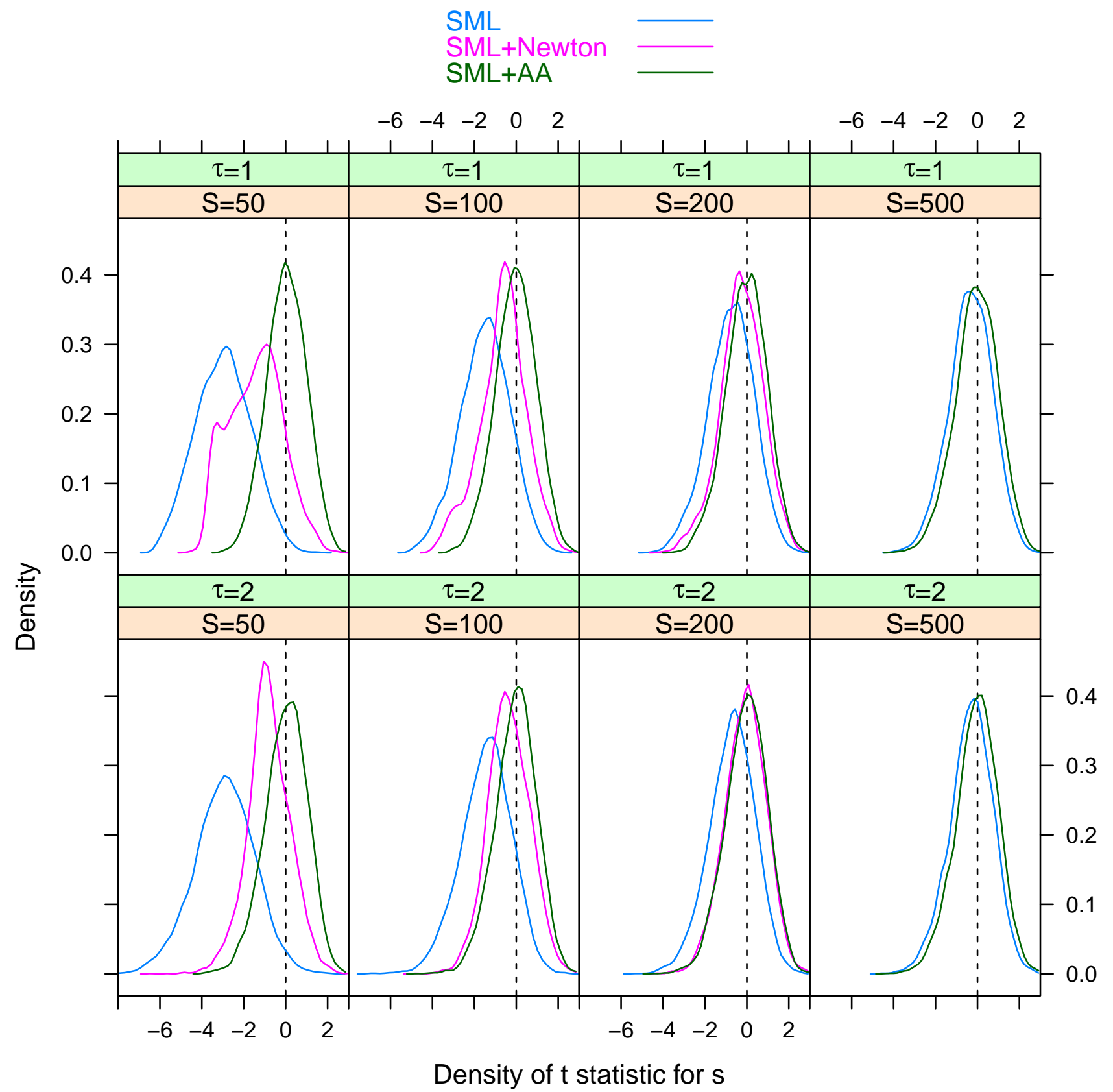

Figure 2: Distributions of the $t$ statistics for $\left(H_{0}\right): s=1$ 
Resorting to one Newton-Raphson step (the "SML+Newton" curves) corrects part of the bias and reduces the variance; but except for large number of simulations, the distribution of the resulting $t$-statistics is still markedly different from $N(0,1)$. Using the AA bias-correction and using the proper formula for the variance-covariance matrix (the "SML+AA" curves), on the other hand, produces distributions that are essentially undistinguishable from $N(0,1)$.

Tables 7 and 8 give the actual coverage probabilities implied by figures 1 and 2. When using uncorrected SML, the nominally $95 \%$ confidence intervals undercover very badly, so that the null hypothesis is rejected up to three-quarters of the time when it is in fact true. Our corrections, on the other hand, yield tests that have close to exact coverage.

\begin{tabular}{|r|r||r|r|r|}
\hline \hline$\tau$ & \multicolumn{1}{|c||}{ S } & SML & SML+Newton & SML+AA \\
\hline \multirow{4}{*}{1} & 50 & 29.5 & 63.1 & 96.1 \\
& 100 & 68.7 & 86.7 & 95.2 \\
& 200 & 85.9 & 92.6 & 94.9 \\
& 500 & 92.7 & - & 94.5 \\
\hline \multirow{4}{*}{2} & 50 & 25.3 & 84.2 & 94.9 \\
& 100 & 69.7 & 93.5 & 95.4 \\
& 200 & 87.2 & 95.4 & 95.6 \\
& 500 & 92.9 & - & 94.6 \\
\hline
\end{tabular}

Table 7: Actual coverage probabilities for $a$

\begin{tabular}{|r|r||r|r|r|}
\hline \hline$\tau$ & \multicolumn{1}{|c||}{ S } & SML & SML+Newton & SML+AA \\
\hline \multirow{4}{*}{1} & 50 & 23.2 & 63.7 & 96.7 \\
& 100 & 66.0 & 87.9 & 96.1 \\
& 200 & 86.6 & 93.3 & 95.6 \\
& 500 & 92.9 & - & 94.3 \\
\hline \multirow{4}{*}{2} & 50 & 23.6 & 87.9 & 95.1 \\
& 100 & 69.0 & 94.0 & 95.3 \\
& 200 & 87.3 & 95.1 & 95.2 \\
& 500 & 93.0 & - & 94.6 \\
\hline
\end{tabular}

Table 8: Actual coverage probabilities for $s$

Like any Monte Carlo study, ours can only be illustrative; yet our results are very encouraging. Our analytical corrections for both bias and variance spectacularly improve inference. Using one Newton step, while less effective, can also be a good way to reduce errors. 


\section{Conclusion}

We developed in this paper a unifying framework for the analysis of approximate estimators. We derived a higher-order expansion of the estimators that takes into account additional biases and variances due to approximations; and we built on this expansion to develop methods that reduce the bias and the efficiency loss that result from the approximation. Simulations on the mixed logit model confirm that the proposed methods work well in finite samples.

We restricted ourselves to estimators where objective function and approximator (as functions of $\theta$ ) were both smooth. In principle, one could import the arguments of Chen et al (2003) to handle non-smooth cases as is done in Armstrong et al (2013). Another approach would be to employ a slight generalization of Robinson (1988, Theorem 1) which in our setting

would yield $\left\|\hat{\theta}_{n, S}-\tilde{\theta}_{n}\right\|=O_{P}\left(\sup _{\left\|\theta-\theta_{0}\right\| \leq \delta}\left\|G_{n}\left(\theta, \hat{\gamma}_{S}\right)-G_{n}(\theta, \gamma)\right\|\right)+o_{P}(1 / \sqrt{n})$, for some $\delta>0$. By strengthening the pointwise bias and variance assumptions to hold uniformly over $\left\|\theta-\theta_{0}\right\| \leq \delta$, we expect our results to remain valid in the non-smooth case. Also, we require the approximators to be mutually independent, which rules out certain recursive approximation schemes such as particle filtering. Establishing results for this more complicated case would be highly useful.

We only allowed for one source of approximation in $\gamma$. More general situations could have several such terms, possibly with quite different properties. This is for example the case in Kristensen and Scherning (2011), which considers the estimation of dynamic discrete choice models: There, one set of simulations are combined with series regression techniques to approximate the value function $\left(\gamma_{1}\right)$, and then another set of simulations are used to compute the conditional choice probabilities $\left(\gamma_{2}\right)$. To cover such situations, Appendix D contains a generalization of Theorem 2 to the case where multiple approximators are employed in the estimation. This is straightforward but tedious, as long as the number of such approximators stays finite; it only requires fairly obvious changes in the assumptions. The expansion can be employed to adjust biases and variances as in the single-approximator case: The analytical bias adjustment will still work when multiple approximators are present, except that we now have to estimate the bias component for each individual approximator. Similarly, the adjustment of standard errors when multiple approximation methods are employed is also relatively straightforward. The Newton-Raphson method would also remain valid. The Jackknife bias adjustment would on the other hand not be easy to extend to the case where biases vanish at different rates.

\section{Acknowledgements}

This paper was previously circulated under the title "Higher-order Improvements of Approximate Estimators". We would like to thank participants at the "Conference on Dynamic 
Aspects in Economic Decision Making" (University of Copenhagen) and seminar audiences at Northwestern, Ohio State, Toulouse, UCL and Yale for helpful comments and suggestions. Kristensen acknowledges financial support from the Economic and Social Research Council through the ESRC Centre for Microdata Methods and Practice grant RES-589-28-0001, the Danish Social Sciences Research Council (through CREATES) and the European Research Council (Starting grant No. 312474). Parts of this research was conducted while Kristensen visited Princeton University and the University of Copenhagen, whose hospitality is gratefully acknowledged. Salanié gratefully acknowledges the Georges Meyer endowment for its support during a visit at the Toulouse School of Economics, as well as the Fundação Getúlio Vargas Rio.

\section{References}

Ackerberg, D., J. Geweke and J. Hahn (2009) Comments on "Convergence Properties of the Likelihood of Computed Dynamic Models". Econometrica 77, 2009-2017.

Altissimo, F. and A. Mele (2009) Simulated Nonparametric Estimation of Dynamic Models. Review of Economic Studies 76, 413-450.

Andrews, D.W.K. (2002) Higher-order Improvements of a Computationally Attractive $k$-step Bootstrap for Extremum Estimators. Econometrica 70, 119-162.

Armstrong, T, Gallant, A. R., Hong, H. and L. Huiyu (2015) The Asymptotic Distribution of Estimators with Overlapping Simulation Draws, mimeo Stanford.

Berry, S., Levinsohn, J., and Pakes, A. (1995) Automobile Prices in Market Equilibrium. Econometrica 63, 841-890.

Bruins, M., Duffy, J., Keane, M. and A. Smith (2015) Generalized Indirect Inference for Discrete Choice Models. Mimeo, Oxford.

Chen, X., O. Linton and I. Van Keilegom (2003) Estimation of Semiparametric Models When the Criterion Function Is Not Smooth. Econometrica 71, 1591-1608.

Creel, M. and D. Kristensen (2012) Estimation of Dynamic Latent Variable Models Using Simulated Nonparametric Moments. Econometrics Journal 15, 490-515.

Dubé, J.P., J. Fox, and C-L. Su (2012) Improving the Numerical Performance of Static and Dynamic Aggregate Discrete Choice Random Coefficients Demand Estimation. Econometrica 80, 2231-2267. 
Duffie, D. and K. J. Singleton (1993) Simulated Moments Estimation of Markov Models of Asset Prices. Econometrica 61, 929-952.

Fermanian, J.-D. and B. Salanié (2004) A Nonparametric Simulated Maximum Likelihood Estimation Method. Econometric Theory 20, 701-734.

Fernández-Villaverde, J. and J.F. Rubio-Ramirez (2005) Estimating Dynamic Equilibrium Economies: Linear versus Nonlinear Likelihood. Journal of Applied Econometrics 20, 891-910.

Fernández-Villaverde, J., J.F. Rubio-Ramirez and M. Santos (2006) Convergence Properties of the Likelihood of Computed Dynamic Models. Econometrica 74, 93-119.

Francq, C. and J.-M. Zakoïan (2005) A Central Limit Theorem for Mixing Triangular Arrays of Variables Whose Dependence Is Allowed to Grow with the Sample Size. Econometric Theory 21, 1165-1171.

Freyberger, J. (2015) Asymptotic Theory for Differentiated Products Demand Models with Many Markets. Journal of Econometrics, 185, 162-181.

Gouriéroux, C. and A. Monfort (1996) Simulation-Based Econometric Methods. Oxford: Oxford University Press.

Hajivassiliou, V.A. (2000) Some Practical Issues in Maximum Simulated Likelihood. In Simulation-based Inference in Econometrics (eds. R. Mariano, T. Schuermann and M.J. Weeks), 71-99. Cambridge: Cambridge University Press.

Hong, H., A. Mahajan and D. Nekipelov (2015) Extremum Estimation and Numerical Derivatives. Journal of Econometrics, forthcoming.

Judd, K., F. F. Kubler and K. Schmedder (2003) Computational Methods for Dynamic Equilibria with Heterogeneous Agents. In Advances in Economics and Econometrics (eds. M. Dewatripont, L.P. Hansen, and S. Turnovsky). Cambridge University Press.

Judd, K. and C. Su (2012) Constrained Optimization Approaches to Estimation of Structural models. Econometrica 80, 2213-2230.

Keane, M. and K. Wolpin (1994) The solution and estimation of discrete choice dynamic programming models by simulation and interpolation: Monte Carlo evidence. The Review of Economics and Statistics 76, 648-672.

Keane, M. and K. Wolpin (1997) The career decisions of young men. Journal of Political Economy, 105, 473-522. 
Kristensen, D. and B. Salanié (2010) Higher Order Improvements for Approximate Estimators. CAM Working Paper 2010-04, University of Copenhagen.

Kristensen, D. and B. Schjerning (2011) Implementation and Estimation of Discrete Markov Decision Models by Sieve Approximations. Manuscript, University of Copenhagen.

Kristensen, D. and Y. Shin (2012) Estimation of Dynamic Models with Nonparametric Simulated Maximum Likelihood. Journal of Econometrics 167, 76-94.

Laffont, J.-J., H. Ossard and Q. Vuong (1995) Econometrics of First-Price Auctions. Econometrica $63,953-980$.

Laroque, G. and B. Salanié (1989) Estimation of Multimarket Fix-Price Models: An Application of Pseudo-maximum Likelihood Methods. Econometrica 57, 831-860.

Lee, L.-F. (1992) On Efficiency of Methods of Simulated Moments and Maximum Simulated Likelihood Estimation of Discrete Response Models. Econometric Theory 8, 518-552.

Lee, L.-F. (1995) Asymptotic Bias in Simulated Maximum Likelihood Estimation of Discrete Choice Models. Econometric Theory 11, 437-483.

Lee, L.-F. (2001) Interpolation, Quadrature, and Stochastic Integration. Econometric Theory 17, 933-961.

McFadden, D.F. (1989) A Method of Simulated Moments for Estimation of Discrete Response Models without Numerical Integration. Econometrica 57, 995-1026.

Newey, W.K. (1991) Kernel Estimation of Partial Means and a General Variance Estimator. Econometric Theory 10, 233-253.

Newey, W.K. and D. McFadden (1994) Large Sample Estimation and Hypothesis Testing. In Handbook of Econometrics, Vol. 4 (eds. R.F. Engle and D.L. McFadden), Chapter 36. Elsevier Science B.V.

Newey, W.K. and R. Smith (2004) Higher-order Properties of GMM and Generalized Empirical Likelihood Estimators. Econometrica 72, 219-255.

Norets, A. (2009) Inference in Dynamic Discrete Choice Models with Serially Correlated Unobserved State Variables. Econometrica 77, 1665-1682.

Norets, A. (2012) Estimation of Dynamic Discrete Choice Models Using Artificial Neural Network Approximations. Econometric Reviews 31, 84-106.

Nze, P.A. and P. Doukhan (2004) Weak Dependence: Models and Applications to Econometrics. Econometric Theory 20, 995-1045. 
Pakes, A. and D. Pollard (1989) Simulation and the Asymptotics of Optimization Estimators. Econometrica 57, 1027-57.

Rio, E. (1994) Inégalités de moments pour les suites stationnaires et fortement mélangeantes. Comptes rendus de l'Académie des Sciences 318, 355-360.

Robinson, P.M. (1988) The Stochastic Difference Between Econometric Statistics. Econometrica $56,531-548$.

Rothenberg, T.J. (1984) Approximating the Distributions of Econometric Estimators and Test Statistics. In Handbook of Econometrics, vol. 2, eds. K. Arrow and M. Intriligator. North Holland.

Rust, J. (1987) Optimal Replacement of GMC Bus Engines: An Empirical Model of Harold Zurcher. Econometrica 55, 999-1033.

Tauchen, G. (1986) Finite State Markov-chain Approximations to Univariate and Vector Autoregressions. Economics Letters 20, 177-181.

Tauchen, G. and R. Hussey (1991) Quadrature-Based Methods for Obtaining Approximate Solutions to Nonlinear Asset Pricing Models. Econometrica 59, 371-396.

Yoshihara, K. (1976) Limiting Behaviour of U-Statistics for Stationary, Absolutely Regular Processes. Zeitschrift für Wahrenscheinlichkeittheorie und verwandte Gebeite 35, 237252.

\section{A Proofs of Main Results}

Proof of Theorem 2. Substituting the expansion given in eq. (6) with $m=2$ into eq. (4), which holds by Lemma 7, yields

$$
\left\|\hat{\theta}_{n, S}-\hat{\theta}_{n}\right\|=O_{P}\left(\left\|\nabla G_{n}\left(\theta_{0}\right)\left[\Delta \hat{\gamma}_{S}\right]+\frac{1}{2} \nabla^{2} G_{n}\left(\theta_{0}\right)\left[\Delta \hat{\gamma}_{S}, \Delta \hat{\gamma}_{S}\right]+R_{n, S}\right\|\right)+o_{P}(1 / \sqrt{n}),
$$

where $\Delta \hat{\gamma}_{i, S}=\hat{\gamma}_{i, S}-\gamma_{0}$. We first derive the rate of the remainder term $R_{n, S}$ :

$$
\begin{aligned}
E\left[\left\|R_{n, S}\right\|\right] & =E\left\|G_{n}\left(\theta_{0}, \hat{\gamma}_{S}\right)-G_{n}\left(\theta_{0}, \gamma_{0}\right)-\nabla G_{n}\left(\theta_{0}\right)\left[\Delta \hat{\gamma}_{S}\right]-\frac{1}{2} \nabla^{2} G_{n}\left(\theta_{0}\right)\left[\Delta \hat{\gamma}_{S}, \Delta \hat{\gamma}_{S}\right]\right\| \\
& \leq \frac{1}{n} \sum_{i=1}^{n} E\left\|g_{i}\left(\theta_{0}, \hat{\gamma}_{i, S}\right)-g_{i}\left(\theta_{0}, \gamma_{0}\right)-\nabla g_{i}\left(\theta_{0}\right)\left[\Delta \hat{\gamma}_{i, S}\right]-\frac{1}{2} \nabla^{2} g_{i}\left(\theta_{0}\right)\left[\Delta \hat{\gamma}_{i, S}, \Delta \hat{\gamma}_{i, S}\right]\right\| \\
& \leq \frac{\bar{G}_{0}}{n} \sum_{i=1}^{n} E\left[\left\|\Delta \hat{\gamma}_{i, S}\right\|^{3}\right],
\end{aligned}
$$


where we have used A.4(2). Applying first Minkowski's inequality and then the inequality $(a+b)^{p} \leq 2^{p-1} a^{p}+2^{p-1} b^{p}$ (which holds for all $a, b>0$ and $p \geq 1$ ), we obtain-dropping the $i$ index:

$$
\begin{aligned}
E\left[\left\|\Delta \hat{\gamma}_{S}\right\|^{3}\right] & =E\left[\left\|\psi_{S}+b_{S}\right\|^{3}\right] \leq\left(E\left[\left\|\psi_{S}\right\|\right]+E\left[\left\|b_{S}\right\|\right]\right)^{3} \leq 4 E\left[\left\|\psi_{S}\right\|^{3}\right]+4 E\left[\left\|b_{S}\right\|^{3}\right] \\
& =O\left(S^{-\alpha_{3}}\right)+O\left(S^{-3 \beta}\right) .
\end{aligned}
$$

The rates of the first and second order functional differentials of $G_{n}\left(\theta_{0}, \gamma\right)$ are given in Lemmas 10 and 11 depending on whether ECA or EIA, as given in eq. (7), is used. These rates together with the rate of $R_{n, S}$ and (26) yield the higher-order stochastic expansion of the EIA and ECA in equation (11). The rates of the leading bias and variance terms as $S \rightarrow \infty$ also follow from Lemmas 10 and 11 .

Finally, the weak convergence of $D_{n, S}$ follows by standard CLT for stationary and mixing triangular arrays. We can, for example, employ Francq and Zakoïan (2005) whose conditions are easily verified given the mixing conditions imposed on data and simulations, which are mutually independent, and the fact that $\operatorname{Var}\left(\sqrt{n}\left\{G_{n}+D_{n, S}\right\}\right)=\Omega_{S}^{G+D}+o(1)=\Omega^{G}+$ $O\left(S^{-\beta}\right)$ where $\Omega^{G}>0$.

Proof of Corollary 3. In the EIA case, $E_{n, S_{n}}=\sum_{i=1}^{n} \nabla g_{i}\left[\psi_{i, S_{n}}\right]$ where $\left(z_{i}, \psi_{i, S_{n}}\right)$, for $i=1, \ldots, n$ and $n \geq 1$, is a stationary and mixing triangular array. Under A.6,

$$
\begin{aligned}
\operatorname{Var}\left(\sqrt{n S_{n}} E_{n, S_{n}}\right) & =\frac{1}{n S_{n}} \sum_{i, j=1}^{n} \sum_{s, t=1}^{S_{n}} E\left[\nabla g_{i}\left[e_{i, s}\right] \nabla g_{j}\left[e_{j, t}\right]\right]=\frac{1}{n S_{n}} \sum_{i=1}^{n} \sum_{s, t=1}^{S_{n}} E\left[\nabla g_{i}\left[e_{i, s}\right] \nabla g_{i}\left[e_{i, t}\right]\right] \\
& =\frac{1}{S_{n}} \sum_{s, t=1}^{S_{n}} E\left[\nabla g_{0}\left[e_{0, s}\right] \nabla g_{0}\left[e_{0, t}\right]\right]=\Omega_{\text {EIA }}^{E}+o(1)
\end{aligned}
$$

where we have used that $E\left[\nabla g_{i}\left[e_{i, s}\right] \nabla g_{j}\left[e_{j, t}\right]\right]=0$ for $i \neq j$, and so the claimed result follows from Francq and Zakoïan (2005). For ECA's satisfying A.6, $\sqrt{S} E_{n, S}=\sum_{s=1}^{S} \nabla G\left\{\tilde{e}_{s}\right] / \sqrt{S}+$ $o_{P}(1) \rightarrow^{d} N\left(0, \Omega_{\mathrm{ECA}}^{E}\right)$, where a CLT for stationary and mixing sequences has been employed.

Proof of Theorem 6. We only give a proof for the case of EIA's; the proof for ECA's follows along the same lines. One can easily show that $\sup _{\theta \in \Theta}\left\|\nabla^{2} \hat{G}_{n}(\theta)\right\|=o_{P}(1)$ as $n, S \rightarrow \infty$, and it now follows by the same arguments as in the proof of Theorem 2 that $\hat{\theta}_{n, S}^{\mathrm{BA}}$ is consistent. Next, we take a Taylor expansion:

$$
o_{P}\left(n^{-1 / 2}\right)=\left\{G_{n}\left(\theta_{0}, \hat{\gamma}_{S}\right)-\frac{1}{2} \nabla^{2} \hat{G}_{n}\left(\theta_{0}\right)\right\}+\left\{H_{n}\left(\bar{\theta}_{n, S}, \hat{\gamma}_{S}\right)-\frac{1}{2} \nabla^{2} \hat{H}_{n}\left(\bar{\theta}_{n, S}\right)\right\}\left(\hat{\theta}_{n, S}^{\mathrm{BA}}-\theta_{0}\right)
$$

where $\nabla^{2} \hat{H}_{n}(\theta)=\partial \nabla^{2} \hat{G}_{n}(\theta) /(\partial \theta)$. From the proof of Theorem $2, H_{n}\left(\bar{\theta}_{n, S}, \hat{\gamma}_{S}\right)=H_{0}+$ 
$o_{P}(1)$, while it is easily shown that $\nabla^{2} \hat{H}_{n}\left(\bar{\theta}_{n, S}\right)=o_{P}(1)$ as $n, S \rightarrow 0$, so that, by the same arguments as in the proof of Theorem 2,

$$
\hat{\theta}_{n, S}^{\mathrm{BA}}-\hat{\theta}_{n}=H_{0}^{-1}\left\{G_{n}\left(\theta_{0}, \hat{\gamma}_{S}\right)-\frac{1}{2} \nabla^{2} \hat{G}_{n}\left(\theta_{0}\right)-G_{n}\left(\theta_{0}, \gamma\right)\right\}+o_{P}(1 / \sqrt{n}) .
$$

Suppressing any dependence on $\theta_{0}$, use (6) to write

$$
\begin{aligned}
G_{n}\left(\hat{\gamma}_{S}\right)-\frac{1}{2} \nabla^{2} \hat{G}_{n}-G_{n}(\gamma)= & \frac{1}{2}\left\{\nabla^{2} G_{n}\left[\psi_{S}, \psi_{S}\right]-\nabla^{2} \hat{G}_{n}\right\}+\nabla G_{n}\left[\hat{\gamma}_{S}-\gamma\right] \\
& +\frac{1}{2}\left\{\nabla^{2} G_{n}\left[\hat{\gamma}_{S}-\gamma, \hat{\gamma}_{S}-\gamma\right]-\nabla^{2} G_{n}\left[\psi_{S}, \psi_{S}\right]\right\}+R_{n, S} .
\end{aligned}
$$

The rates of the second and third terms of (27) are derived in Lemma 11. To ensure that $R_{n, S}$ is negligible, we build on Lemma 12, which uses A.6 to deliver a better rate than that obtained in the proof of Theorem 2 .

The crucial term is the first term of (27). Recall $\hat{\gamma}_{i}(x)=S^{-1} \sum_{s=1}^{S} w_{i s}(x)$, and the definition of $\nabla^{2} \hat{G}_{n}$ in eq. (17). Using the bilinearity of $\left(d \gamma, d \gamma^{\prime}\right) \mapsto \nabla^{2} g_{i}\left[d \gamma, d \gamma^{\prime}\right]$, and denoting $\bar{w}_{i}(x)=E\left[w_{i, s}(x)\right]$ and $e_{i s}(x)=w_{i s}(x)-\bar{w}_{i}(x)$,

$$
\begin{aligned}
& \nabla^{2} G_{n}\left[\psi_{n, S}, \psi_{n, S}\right]-\nabla^{2} \hat{G}_{n} \\
= & \frac{1}{n S^{2}} \sum_{i=1}^{n} \sum_{s \neq t} \nabla^{2} g_{i}\left[e_{i s}, e_{i t}\right]+\frac{1}{n S^{2}} \sum_{i=1}^{n} \sum_{s=1}^{S} \nabla^{2} g_{i}\left[e_{i s}, e_{i s}\right]-\frac{1}{n S^{2}} \sum_{i=1}^{n} \sum_{s=1}^{S} \nabla g_{i}\left[w_{i s}-\hat{\gamma}_{i}, w_{i s}-\hat{\gamma}_{i}\right] \\
= & \frac{1}{n S^{2}} \sum_{i=1}^{n} \sum_{s \neq t} \nabla^{2} g_{i}\left[e_{i s}, e_{i t}\right]+\frac{1}{n S^{2}} \sum_{i=1}^{n} \sum_{s=1}^{S}\left\{\nabla^{2} g_{i}\left[e_{i s}, e_{i s}\right]-\nabla g_{i}\left[w_{i s}-\hat{\gamma}_{i}, w_{i s}-\hat{\gamma}_{i}\right]\right\} \\
= & \frac{1}{n S^{2}} \sum_{i=1}^{n} \sum_{s \neq t} \nabla^{2} g_{i}\left[e_{i s}, e_{i t}\right]+\frac{1}{n S^{2}} \sum_{i=1}^{n} \sum_{s=1}^{S}\left\{\nabla^{2} g_{i}\left[\hat{\gamma}_{i}-\bar{w}_{i}, e_{i s}\right]+\nabla^{2} g_{i}\left[e_{i s}, \hat{\gamma}_{i}-\bar{w}_{i}\right]\right\} \\
= & \frac{1}{n S^{2}} \sum_{i=1}^{n} \sum_{s \neq t} \nabla^{2} g_{i}\left[e_{i s}, e_{i t}\right]+\frac{2}{n S} \sum_{i=1}^{n} \nabla^{2} g_{i}\left[\hat{\gamma}_{i}-\bar{w}_{i}, \hat{\gamma}_{i}-\bar{w}_{i}\right],
\end{aligned}
$$

where the last equality uses the fact that $S^{-1} \sum_{s=1}^{S} e_{i s}=\hat{\gamma}_{i}-\bar{w}_{i}$. Start with the first term, and note that $E\left[\nabla^{2} g_{i}\left[e_{i s}, e_{i t}\right]\right]=0$ when $s \neq t$. Then apply Lemma 8 with $r=1$ to $W_{i, S}:=S^{-2} \sum_{s \neq t} \nabla^{2} g_{i}\left[e_{i s}, e_{i t}\right]$, getting

$$
\operatorname{Var}\left(\frac{1}{2 n S^{2}} \sum_{i=1}^{n} \sum_{s \neq t} \nabla^{2} g_{i}\left[e_{i s}, e_{i t}\right]\right) \leq \frac{C}{n} E\left[\left\|W_{i, S}\right\|^{2+\delta}\right]^{2 /(2+\delta)} .
$$

Now $W_{i, S}$ is a degenerate $U$-statistic since $E\left[\nabla^{2} g\left(z_{i}\right)\left[e_{i s}, e_{i t}\right] \mid z_{i}, e_{i t}\right]=E\left[\nabla^{2} g\left(z_{i}\right)\left[e_{i s}, e_{i t}\right] \mid z_{i}, e_{i s}\right]=$ 0 . Given the conditions imposed on $\left\{e_{i, s}: 1 \leq s \leq S\right\}$ in (A.6), we can employ $U$-statistic re- 
sults for absolutely regular sequences: Yoshihara (1976, Lemma 3) states that $E\left[\left\|W_{i, S}\right\|^{4} \mid z_{i}\right]=$ $O\left(S^{-4}\right)$. By inspection of the proof of Yoshihara (1976, Lemma 3), it is easily checked that in fact, for some constant $C>0$ we have $E\left[\left\|W_{i, S}\right\|^{4} \mid z_{i}\right] \leq C S^{-4} M_{S}\left(z_{i}\right)$, where $M_{S}\left(z_{i}\right):=\sup _{s<t} E\left[\left\|\nabla^{2} g\left(z_{i}\right)\left[e_{i s}, e_{i t}\right]\right\|^{4+\epsilon} \mid z_{i}\right]^{4 /(4+\epsilon)}$, for some $\epsilon>0$. Thus, with $\delta=2$ and using the Lipschitz condition on $\nabla^{2} g$, we obtain

$$
\begin{aligned}
E\left[\left\|W_{i, S}\right\|^{4}\right] & \leq C S^{-4} E\left[M_{S}\left(z_{i}\right)\right] \\
& \leq C S^{-4} E\left[\sup _{s<t} E\left[\left\|\nabla^{2} g\left(z_{i}\right)\left[e_{i s}, e_{i t}\right]\right\|^{4+\epsilon} \mid z_{i}\right]^{4 /(4+\epsilon)}\right] \\
& \left.\left.\leq C S^{-4} E\left[b^{4}\left(z_{i}\right) \sup _{s<t} E\left[\| e_{i s}(z)\right]\left\|^{4+\epsilon}\right\| e_{i t}(z)\right] \|^{4+\epsilon} \mid z_{i}\right]^{4 /(4+\epsilon)}\right] \\
& \left.\leq C S^{-4} E\left[b^{4}\left(z_{i}\right) E\left[\| e_{i s}(z)\right] \|^{8+\epsilon} \mid z_{i}\right]^{4 /(8+\epsilon)}\right] \\
& \leq C S^{-4} \sqrt{E\left[b^{8}\left(z_{i}\right)\right]} E\left[\left\|e_{i s}\right\|^{8+2 \epsilon}\right]^{4 /(8+2 \epsilon)} \\
& =O\left(S^{-4+\mu_{8} / 2}\right) .
\end{aligned}
$$

It follows that $\sum_{i=1}^{n} \sum_{s \neq t} \nabla^{2} g_{i}\left[e_{i s}, e_{i t}\right] /\left(n S^{2}\right)=O_{P}\left(n^{-1 / 2} S^{-1+\mu_{8} / 4}\right)$. As for the second term, by definition $\hat{\gamma}_{i}-\bar{w}_{i}=\psi_{i, S}$; and it follows from Lemma 9 that $E\left[\nabla^{2} g_{i}\left[\psi_{i, S}, \psi_{i, S}\right]\right]=$ $O\left(S^{-\alpha_{2}}\right)$ and $\frac{1}{n} \sum_{i=1}^{n}\left(\nabla^{2} g_{i}\left[\psi_{i, S}, \psi_{i, S}\right]-E\left[\nabla^{2} g_{i}\left[\psi_{i, S}, \psi_{i, S}\right]\right]\right)=O_{P}\left(n^{-1 / 2} S^{-\alpha_{4} / 2}\right)$. Summing up, $\tilde{B}_{2}=H_{0}^{-1} E\left[\nabla^{2} G_{n}\left[\psi_{n, S}, \psi_{n, S}\right]-\nabla^{2} \hat{G}_{n}\right] / 2=O\left(S^{-2+\mu_{2}}\right)$ while

$$
\operatorname{Var}\left(\nabla^{2} G_{n}\left[\psi_{n, S}, \psi_{n, S}\right]-\nabla^{2} \hat{G}_{n}\right)=O\left(n^{-1} S^{-2+\mu_{8} / 2}\right)+O\left(n^{-1} S^{-2+\alpha_{4}}\right) .
$$

This completes the proof.

Proof of Theorem 5. To apply the general result in Robinson (1988, Theorem 2), we need to check that his conditions A.1 and A.3 are satisfied in our application. His condition A.1 requires consistency of the approximate estimator for a suitable choice of $S$, which our assumptions imply. Robinson's condition A.3 also holds given the smoothness conditions imposed on $G_{n}\left(\theta, \hat{\gamma}_{S}\right)$ in our Assumption A.2.

\section{B Lemmas}

Lemma 7 Under Assumptions A.1-A.3, eq. (4) holds. 
Proof. We first take a Taylor expansion of $G_{n}\left(\theta, \gamma_{0}\right)$ and $G_{n}\left(\theta, \hat{\gamma}_{S}\right)$ w.r.t. $\theta$ :

$$
\begin{aligned}
& o_{P}\left(n^{-1 / 2}\right)=G_{n}\left(\hat{\theta}_{n}, \gamma_{0}\right)=G_{n}\left(\theta_{0}, \gamma_{0}\right)+H_{n}\left(\bar{\theta}_{n}, \gamma_{0}\right)\left(\hat{\theta}_{n}-\theta_{0}\right) \\
& o_{P}\left(n^{-1 / 2}\right)=G_{n}\left(\hat{\theta}_{n, S}, \hat{\gamma}_{S}\right)=G_{n}\left(\theta_{0}, \hat{\gamma}_{S}\right)+H_{n}\left(\tilde{\theta}_{n, S}, \hat{\gamma}_{S}\right)\left(\hat{\theta}_{n, S}-\theta_{0}\right)
\end{aligned}
$$

for some $\bar{\theta}_{n}\left(\tilde{\theta}_{n, S}\right)$ between $\hat{\theta}_{n}\left(\hat{\theta}_{n, S}\right)$ and $\theta_{0}$. Since $\hat{\theta}_{n}\left(\hat{\theta}_{n, S}\right)$ is consistent, $\bar{\theta}_{n, S}\left(\tilde{\theta}_{n, S}\right) \stackrel{P}{\rightarrow} \theta_{0}$. By standard arguments together with Assumption A.2,

$$
\begin{aligned}
\left\|H_{n}\left(\tilde{\theta}_{n, S}, \hat{\gamma}_{S}\right)-H_{0}\right\| \leq & \left\|H_{n}\left(\tilde{\theta}_{n, S}, \hat{\gamma}_{S}\right)-H_{n}\left(\tilde{\theta}_{n, S}, \gamma_{0}\right)\right\|+\left\|H_{n}\left(\tilde{\theta}_{n, S}, \gamma_{0}\right)-H\left(\tilde{\theta}_{n, S}, \gamma_{0}\right)\right\| \\
& +\left\|H\left(\tilde{\theta}_{n, S}, \gamma_{0}\right)-H\left(\theta_{0}, \gamma_{0}\right)\right\| \\
\leq & \sup _{\left\|\theta-\theta_{0}\right\| \leq \delta}\left\|H_{n}\left(\theta, \hat{\gamma}_{S}\right)-H_{n}\left(\theta, \gamma_{0}\right)\right\|+\sup _{\left\|\theta-\theta_{0}\right\| \leq \delta}\left\|H_{n}\left(\theta, \gamma_{0}\right)-H\left(\theta, \gamma_{0}\right)\right\| \\
& +\left\|H\left(\tilde{\theta}_{n, S}, \gamma_{0}\right)-H\left(\theta_{0}, \gamma_{0}\right)\right\| \\
= & o_{P}(1),
\end{aligned}
$$

and similar for $H_{n}\left(\bar{\theta}_{n}, \gamma_{0}\right)$. Going back to eqs. (28)-(29), we have now shown that

$$
\hat{\theta}_{n, S}-\theta_{0}=-H_{0}^{-1} G_{n}\left(\theta_{0}, \hat{\gamma}_{S}\right)+o_{P}(1 / \sqrt{n}), \quad \hat{\theta}_{n}-\theta_{0}=-H_{0}^{-1} G_{n}\left(\theta_{0}, \gamma_{0}\right)+o_{P}(1 / \sqrt{n}) .
$$

Subtracting the second expansion from the first gives the result.

The following auxiliary results will be used in the analysis of the first and second order differentials:

Lemma 8 Let $\left\{W_{i}\right\}$ be a sequence of $\alpha$-mixing random variables with $E\left[W_{i}\right]=0, E\left[\left\|W_{i}\right\|^{2 r+\delta}\right]<$ $\infty$ for some $r \geq 1$ and $\delta>0$ and its mixing coefficients $\alpha_{i}, i=1,2, \ldots$, satisfying $\alpha_{i} \leq A i^{-a}$ for some $A>0$, and $a>2 r+4 r(r-1) / \delta-2$. Then there exists a constant $C=C(r, a, A)<$ $\infty$ such that:

$$
E\left[\left\|\frac{1}{n} \sum_{i=1}^{n} W_{i}\right\|^{2 r}\right] \leq n^{-r} \times C E\left[\left\|W_{i}\right\|^{2+\delta}\right]^{2 r /(2+\delta)}+o\left(n^{-r}\right) .
$$

Proof. From Rio (1994), we have for $r \geq 1$,

$$
E\left[\left\|\frac{1}{n} \sum_{i=1}^{n} W_{i}\right\|^{2 r}\right] \leq C_{r}\left[n^{-r} M_{2, \alpha, n}^{r}+n^{1-2 r} M_{2 r, \alpha, n}\right],
$$

where the numbers $M_{p, \alpha, n}$ are defined in Rio (1994). By Nze and Doukhan (2004, p. 1040),

$$
M_{p, \alpha, n} \leq\left[E\left\|W_{i}\right\|^{p+\delta}\right]^{p /(p+\delta)} \times \frac{(p+\delta)(p-1)}{\delta} \sum_{n=0}^{\infty}(n+1)^{p+p(p-1) / \delta-2} \alpha_{n} .
$$


Given the bound we imposed on the mixing coefficients, there exists a constant $C(A, a)$ such that

$$
\sum_{n=0}^{\infty}(n+1)^{p+p(p-1) / \delta-2} \alpha_{n} \leq C(A, a) \sum_{n=0}^{\infty}(n+1)^{p+p(p-1) / \delta-2-a}<\infty .
$$

In particular, there exist constants $C(r, A, a)$ such that

$$
M_{2, \alpha, n}^{r} \leq C(r, A, a)\left[E\left\|W_{i}\right\|^{2+\delta}\right]^{2 r /(2+\delta)}, \text { and } M_{2 r, \alpha, n} \leq C(r, A, a)\left[E\left\|W_{i}\right\|^{2 r+\delta}\right]^{2 r /(2 r+\delta)} .
$$

The result follows by noting that $n^{1-2 r}=o\left(n^{-r}\right)$ for $r>1$, and that for $r=1$ both terms in equation (30) are of order $n^{-1}=n^{-r}$.

Lemma 9 Assume that $\left\{z_{i}\right\}$ satisfies Assumption A.1, and that $\hat{\gamma}_{i, S}$ satisfy Assumption A.5(4) for $i=1, \ldots, J$. Let $m(z ; d \gamma)$ be a functional satisfying:

$$
E\left[\|m(z ; d \gamma)\|^{2 r+\delta}\right]<\infty, \quad E\left[\|m(z ; d \gamma)\|^{2+\delta}\right] \leq \bar{M}\|d \gamma\|^{k(2+\delta)},
$$

for some $r, k \geq 1$ and $\delta>0$. Then, with $b_{S}$ and $\psi_{S}$ given in A.5, the following hold:

(i) For EIA's, with $M_{S}^{V}:=E\left[m\left(z_{i} ; \psi_{i, S}\right)\right]$ and $M_{S}^{B}:=E\left[m\left(z_{i} ; b_{i, S}\right)\right]$,

$$
\begin{gathered}
E\left[\left\|\frac{1}{n} \sum_{i=1}^{n}\left\{m\left(z_{i} ; b_{i, S}\right)-M_{S}^{B}\right\}\right\|^{2 r}\right]=O\left(n^{-r}\right) \times\left[E\left\|b_{S}\right\|^{k(2+\delta)}\right]^{2 r /(2+\delta)}, \\
E\left[\left\|\frac{1}{n} \sum_{i=1}^{n}\left\{m\left(z_{i} ; \psi_{i, S}\right)-M_{S}^{V}\right\}\right\|^{2 r}\right]=O\left(n^{-r}\right) \times\left[E\left\|\psi_{S}\right\|^{k(2+\delta)}\right]^{2 r /(2+\delta)} .
\end{gathered}
$$

(ii) For $E C A$ 's, with $\bar{m}(\gamma)=E[m(z ; \gamma)]$ for any fixed $\gamma$,

$$
\begin{gathered}
E\left[\left\|\frac{1}{n} \sum_{i=1}^{n}\left\{m\left(z_{i} ; b_{S}\right)-\bar{m}\left(b_{S}\right)\right\}\right\|^{2 r}\right]=O\left(n^{-r}\right) \times\left[E\left\|\psi_{S}\right\|^{k(2+\delta)}\right]^{2 r /(2+\delta)}, \\
E\left[\left\|\frac{1}{n} \sum_{i=1}^{n}\left\{m\left(z_{i} ; \psi_{S}\right)-\bar{m}\left(\psi_{S}\right)\right\}\right\|^{2 r}\right]=O\left(n^{-r}\right) \times\left[E\left\|\psi_{S}\right\|^{k(2+\delta)}\right]^{2 r /(2+\delta)} .
\end{gathered}
$$

(iii) The means satisfy $\left\|M_{S}^{B}\right\| \leq \bar{M} E\left[\left\|b_{i, S}\right\|^{k}\right],\left\|M_{S}^{V}\right\| \leq \bar{M} E\left[\left\|\psi_{i, S}\right\|^{k}\right]$, and $E\left[\left\|\bar{m}\left(\psi_{S}\right)\right\|^{2 r}\right] \leq$ $\bar{M} E\left[\left\|\psi_{S}\right\|^{2 k r}\right]$.

Proof. Define $W_{i, S}:=m\left(z_{i} ; \psi_{i, S}\right)-M_{S}\left(\psi_{i, S}\right)$. By assumptions (A.1) and (A.5), $\left\{W_{i, S}\right\}$ is a geometrically mixing process for any given value of $S$ and so its mixing coefficients satisfy the mixing conditions imposed in Lemma 8. Furthermore, (32) implies that $E\left[\left\|W_{i, S}\right\|^{2 r+\delta}\right]<\infty$. 
We can therefore apply Lemma 8

$E\left[\left\|\frac{1}{n} \sum_{i=1}^{n}\left\{m\left(z_{i} ; \psi_{i, S}\right)-M_{S}\left(\psi_{i, S}\right)\right\}\right\|^{2 r}\right] \leq C n^{-r}\left[E\left\|m\left(z_{i} ; \psi_{i, S}\right)-M_{S}\left(\psi_{i, S}\right)\right\|^{2+\delta}\right]^{2 r /(2+\delta)}+o\left(n^{-r}\right)$

where $C=C(r, a, A)$ only depends on $r$ and the mixing coefficients of $\left\{z_{i}\right\}$ and $\left\{\psi_{i, S}\right\}$. By (32), $E\left[\left\|m\left(z ; \psi_{i, S}\right)\right\|^{2+\delta}\right] \leq \bar{M} E\left[\left\|\psi_{i, S}\right\|^{k(2+\delta)}\right] n^{-r}$ and $\left\|M_{S}\left(\psi_{i, S}\right)\right\| \leq E\left[\left\|m\left(z_{i} ; \psi_{i, S}\right)\right\|\right] \leq$ $\bar{M} E\left[\left\|\psi_{i, S}\right\|^{k}\right]$. It is easily seen that the above inequalities still go through when replacing $\psi_{i, S}$ with $b_{i, S}$. This prove (i) and (iii).

To derive the second inequality of (ii), now redefine $W_{i, S}$ as $W_{i, S}:=m\left(z_{i} ; \psi_{S}\right)-\bar{m}\left(\psi_{S}\right)$. It is easily seen that conditionally on $\psi_{S},\left(W_{i, S}\right)$ satisfies the conditions of Lemma 8 , so that

$$
E\left[\left\|\frac{1}{n} \sum_{i=1}^{n} W_{i, S}\right\|^{2 r} \mid \psi_{S}\right] \leq C E\left[\left\|W_{i, S}\right\|^{2+\delta} \mid \psi_{S}\right] n^{-r}+o\left(n^{-r}\right),
$$

where $C=C(r, a, A)$ does not depend on $\psi_{S}$. Next, observe that

$$
E\left[\left\|W_{i, S}\right\|^{2+\delta}\right] \leq C E\left[\left\|m\left(z ; \psi_{S}\right)\right\|^{2+\delta}\right] \leq C \bar{M} E\left[\left\|\psi_{S}\right\|^{k(2+\delta)}\right]
$$

we conclude that

$E\left[\left\|\frac{1}{n} \sum_{i=1}^{n} W_{i, S}\right\|^{2 r}\right]=E\left[E\left[\left\|\frac{1}{n} \sum_{i=1}^{n} W_{i, S}\right\|^{2 r} \mid \psi_{S}\right]\right] \leq C E\left[\left\|\psi_{S}\right\|^{k(2+\delta)}\right] n^{-r}+o\left(n^{-r}\right)$.

Finally, $E\left[\left\|\bar{m}\left(\psi_{S}\right)\right\|^{2 r}\right] \leq E\left[\left\|m\left(z ; \psi_{S}\right)\right\|^{2 r}\right] \leq \bar{M} E\left[\left\|\psi_{S}\right\|^{2 r k}\right]$. The proof of the first inequality of (ii) follows along the same lines.

In the next three lemmas, dependence on $\theta$ is suppressed since it is kept fixed at $\theta_{0}$.

Lemma 10 Under A.1-A.3, A.4(2), and A.6(4), the first and second order differentials of $G_{n}\left(\theta_{0}, \hat{\gamma}_{S}\right)$ for the ECA yield the rates given in Theorem 2.

Proof. For ECA, the functional differentials of $G_{n}$ are given by

$$
\nabla G_{n}[d \gamma]=\frac{1}{n} \sum_{i=1}^{n} \nabla g_{i}[d \gamma], \quad \nabla^{2} G_{n}\left[d \gamma, d \gamma^{\prime}\right]=\frac{1}{n} \sum_{i=1}^{n} \nabla^{2} g_{i}\left[d \gamma, d \gamma^{\prime}\right]
$$

and $d \gamma$ and $d \gamma^{\prime}$ are the same for all observations $i=1, \ldots, n$. Given A.6(4), the application of the first-order differential to the bias component can be rewritten as

$$
\nabla G_{n}\left[b_{S}\right]=S^{-\beta} \frac{1}{n} \sum_{i=1}^{n} \nabla g_{i}[\bar{b}]+\frac{1}{n} \sum_{i=1}^{n} \nabla g_{i}\left[b_{S}-S^{-\beta} \bar{b}\right] \text {. }
$$


Now, $E\left[\sum_{i=1}^{n} \nabla g_{i}[\bar{b}] / n\right]=E\left[\nabla g_{i}[\bar{b}]\right]$, and

$$
E\left[\frac{1}{n} \sum_{i=1}^{n}\left\|\nabla g_{i}\left[b_{S}-S^{-\beta} \bar{b}\right]\right\|\right] \leq G_{1}\left\|b_{S}-S^{-\beta} \bar{b}\right\|=o\left(S^{-\beta}\right) .
$$

By Lemma 9(i) with $m(z ; d \gamma)=\nabla g(z)[d \gamma], k=1$ and $r=1$, $\operatorname{Var}\left(\nabla G_{n}\left[b_{S}\right]\right) \leq \frac{1}{n} C\left\|b_{S}\right\|^{2}=$ $O\left(S^{-2 \beta} / n\right)$. Since $d \gamma \mapsto \nabla g_{i}[d \gamma]$ is linear, the conditional mean of the stochastic component of the first-order term is $E\left[\nabla G_{n}\left[\psi_{S}\right] \mid \mathcal{Z}_{n}\right]=\frac{1}{n} \sum_{i=1}^{n} \nabla g_{i}\left[E\left[\psi_{S} \mid z_{i}\right]\right]=0$. Moreover, define $\nabla G[\gamma]=E\left[\nabla g_{i}[\gamma]\right]$ (where expectations are taken w.r.t. the observation $z_{i}$ ); then $\nabla G_{n}\left[\psi_{S}\right]=\nabla G\left[\psi_{S}\right]+\frac{1}{n} \sum_{i=1}^{n}\left\{\nabla g_{i}\left[\psi_{S}\right]-\nabla G\left[\psi_{S}\right]\right\}$. Recalling the definition of $\nabla G\left[\psi_{S}\right]$, it follows from Lemma 9 (ii) with $m(z ; d \gamma)=\nabla g(z)[d \gamma]$ and $k=2$ that the first term satisfies $\operatorname{Var}\left(\nabla G\left[\psi_{S}\right]\right) \leq M E\left[\left\|\psi_{S}\right\|^{2}\right]=O\left(S^{-\alpha_{2}}\right)$ while the second term is $O_{P}\left(n^{-1 / 2} S^{-\alpha_{2}}\right)$.

Regarding the second order differential, its application to the bias component satisfies

$$
\nabla^{2} G_{n}\left[b_{S}, b_{S}\right]=S^{-2 \beta} \frac{1}{n} \sum_{i=1}^{n} \nabla^{2} g_{i}[\bar{b}, \bar{b}]+o_{P}\left(S^{-2 \beta}\right)
$$

moreover, $E\left[\sum_{i=1}^{n} \nabla^{2} g_{i}[\bar{b}, \bar{b}] / n\right]=E\left[\nabla^{2} g_{i}[\bar{b}, \bar{b}]\right]$ and, applying Lemma 9(ii) with $m(z ; d \gamma)=$ $\nabla^{2} g(z)[d \gamma, d \gamma], k=2$ and $r=1, \operatorname{Var}\left(\nabla^{2} G_{n}\left[b_{S}, b_{S}\right]\right) \leq \frac{1}{n} C\left\|b_{S}\right\|^{4}=O\left(n^{-1} S^{-4 \beta}\right)$. To bound the variance component, define $\nabla^{2} G[\gamma, \gamma]=E\left[\nabla^{2} g_{i}[\gamma, \gamma]\right]$, and write

$$
\nabla^{2} G_{n}\left[\psi_{S}, \psi_{S}\right]=\nabla^{2} G\left[\psi_{S}, \psi_{S}\right]+\frac{1}{n} \sum_{i=1}^{n}\left(\nabla^{2} g_{i}\left[\psi_{S}, \psi_{S}\right]-\nabla^{2} G\left[\psi_{S}, \psi_{S}\right]\right)
$$

Applying Lemma 9(ii) with $m(z ; d \gamma)=\nabla^{2} g(z)[d \gamma, d \gamma]$ and $r=1, k=2$, we obtain that $E\left\|\nabla^{2} G_{n}\left[\psi_{S}, \psi_{S}\right]\right\|=O_{P}\left(S^{-2 \alpha_{2}}\right)$.

Finally, by the same arguments as before, $E\left[\nabla^{2} G_{n}\left[\psi_{S}, b_{S}\right]\right]=0$ while $\operatorname{Var}\left(\nabla^{2} G_{n}\left[\psi_{S}, b_{S}\right]\right)=$ $O\left(n^{-1} S^{-\alpha_{4}}\right)$ and $\operatorname{Var}\left(\nabla^{2} G_{n}\left[\psi_{S}, b_{S}\right]\right)=O\left(n^{-1} S^{-\alpha_{2}-2 \beta}\right)$.

Lemma 11 Under A.1-A.3, A.4(2) and A.5(4), the first and second order differentials of $G_{n}\left(\theta_{0}, \gamma_{S}\right)$ for the EIA in (7) yield the rates given in Theorem 2.

Proof. For the EIA, the first and second order differentials are $\nabla G_{n}[d \gamma]=\sum_{i=1}^{n} \nabla g_{i}\left[d \gamma_{i}\right] / n$ and $\left.\nabla^{2} G_{n}\right)\left[d \gamma, d \gamma^{\prime}\right]=\sum_{i=1}^{n} \nabla^{2} g_{i}\left[d \gamma_{i}, d \gamma_{i}^{\prime}\right] / n$, for any $d \gamma=\left(d \gamma_{1}, \ldots, d \gamma_{n}\right)$ and $d \gamma^{\prime}=\left(d \gamma_{1}^{\prime}, \ldots, d \gamma_{n}^{\prime}\right)$. It is easily seen that the bias components are the same as those we derived for the ECA in Lemma 10, and so we only consider the variance components. With $\mathcal{Z}_{n}=\left(z_{1}, \ldots, z_{n}\right)$, the mean of the first-order variance component is zero, $E\left[\nabla G_{n}\left[\psi_{S}\right] \mid \mathcal{Z}_{n}\right]=\sum_{i=1}^{n} \nabla g_{i}\left[E\left[\psi_{i, S} \mid z_{i}\right]\right] / n=$ 0 , while its variance satisfies, using Lemma 9.(i) with $m(z, \gamma)=\nabla g(z)[\gamma]$ (in particular, $\left.M_{S}^{V}=0\right), \operatorname{Var}\left(\nabla G_{n}\left[\psi_{S}\right]\right) \leq \frac{1}{n} C E\left[\left\|\psi_{S}\right\|^{2}\right]=O\left(n^{-1} S^{-\alpha_{2}}\right)$. Applying Lemma 9(i) and (iii) 
with $m(z ; d \gamma)=\nabla^{2} g(z)[d \gamma, d \gamma]$ and $k=2$, the mean and the variance of the second order differential satisfy

$$
E\left[\nabla^{2} G_{n}\left[\psi_{S}, \psi_{S}\right]\right]=E\left[\nabla^{2} g_{i}\left[\psi_{i, S}, \psi_{i, S}\right]\right] \leq C E\left[\left\|\psi_{i, S}\right\|^{2}\right]=O\left(S^{-\alpha_{2}}\right)
$$

and $\operatorname{Var}\left[\nabla^{2} G_{n}\left[\psi_{S}, \psi_{S}\right]\right]=O\left(n^{-1} S^{-\alpha_{4}}\right)$. The cross term satisfies $E\left[\nabla^{2} G_{n}\left[\psi_{S}, b_{S}\right]\right]=0$ while $\operatorname{Var}\left(\nabla^{2} G_{n}\left[\psi_{S}, b_{S}\right]\right)=O\left(n^{-1} S^{-\alpha_{2}} S^{-2 \beta}\right)$, and so we can ignore this term since it is of lower order.

Lemma 12 Assume that A.1-A.3, A.4(3) and A.6(6) hold. Then the rate of the remainder term $R_{n, S}$ can be sharpened to:

$$
R_{n, S}=O_{P}\left(S^{-3 \beta}\right)+O_{P}\left(S^{-\left(2-\mu_{4}\right)}\right)+O\left(S^{-\left(2-\mu_{3}\right)}\right)+O\left(n^{-1 / 2} S^{-\left(3-\mu_{6}\right) / 2}\right) .
$$

Proof. Since the third-order differential exists, the remainder term in (6) can be further expanded to obtain $R_{n, S}=\nabla^{3} G_{n}\left[\Delta \hat{\gamma}_{S}, \Delta \hat{\gamma}_{S}, \Delta \hat{\gamma}_{S}\right] / 6+\bar{R}_{n, S}$ where, by A.4(3) and the same arguments used in the proof of Theorem 2, $E\left[\left\|\bar{R}_{n, S}\right\|\right] \leq \bar{G}_{0} E\left[\left\|\Delta \hat{\gamma}_{i, S}\right\|^{4}\right]=O\left(S^{-4 \beta}\right)+$ $O\left(S^{-\left(2-\mu_{4}\right)}\right)$. Regarding the third order term, it is easy to check that the bias component is of order $O_{P}\left(S^{-3 \beta}\right)+O_{P}\left(n^{-1 / 2} S^{-3 \beta}\right)$, by arguments similar to those used in Lemma 10 .

This leaves the variance component. In the case of EIA, the variance component can be written as $\nabla^{3} G_{n}\left[\psi_{S}, \psi_{S}, \psi_{S}\right]=\sum_{i=1}^{n} \nabla^{3} g_{i}\left[\psi_{S}, \psi_{S}, \psi_{S}\right] / n$. By Lemma 9 , we obtain:

$$
\nabla^{3} G_{n}\left[\psi_{S}, \psi_{S}, \psi_{S}\right]-E\left[\nabla^{3} G_{n}\left[\psi_{S}, \psi_{S}, \psi_{S}\right]\right]=O\left(n^{-1 / 2} S^{-\left(3-\mu_{6}\right) / 2}\right)
$$

given the independence between simulations,

$$
\begin{aligned}
\left|E\left[\nabla^{3} G_{n}\left[\psi_{S}, \psi_{S}, \psi_{S}\right]\right]\right| & \leq \frac{1}{S^{3}} \sum_{s, t, u=1}^{S}\left|E\left[\nabla^{3} g_{i}\left[e_{i, s}, e_{i, t}, e_{i, u}\right]\right]\right|=\frac{\left|E\left[\nabla^{3} g_{i}\left[e_{i, s}, e_{i, s}, e_{i, s}\right]\right]\right|}{S^{2}} \\
& \leq \frac{C}{S^{2}} E\left[e_{i, s}^{3}\right]=O\left(S^{-\left(2-\mu_{3}\right)}\right) .
\end{aligned}
$$

In the case of ECA, define $\nabla^{3} \bar{g}[\gamma, \gamma, \gamma]=E\left[\nabla^{2} g_{i}[\gamma, \gamma, \gamma]\right]$ and write

$$
\nabla^{3} G_{n}\left[\psi_{S}, \psi_{S}, \psi_{S}\right]=\nabla^{3} \bar{g}\left[\psi_{S}, \psi_{S}, \psi_{S}\right]+\frac{1}{n} \sum_{i=1}^{n}\left\{\nabla^{3} g_{i}\left[\psi_{S}, \psi_{S}, \psi_{S}\right]-\nabla^{3} \bar{g}\left[\psi_{S}, \psi_{S}, \psi_{S}\right]\right\}
$$

Applying Lemma 9.(ii) with $m(z ; d \gamma)=\nabla^{3} g(z)[d \gamma, d \gamma, d \gamma]$, the two terms are $O_{P}\left(S^{-\left(3 / 2-\mu_{3}\right)}\right)$ and $O_{P}\left(n^{-1 / 2} S^{-\left(3-\mu_{6}\right) / 2}\right)$ respectively. 


\section{Details on Applications}

\section{C.1 Expansion of NPSMLE}

NPSMLE is identical to SMLE except that $p\left(=\gamma_{0}\right)$ is approximated by a kernel density estimator based on simulated $y_{s}$ 's, $\hat{p}_{S}(y, x ; \theta)=\sum_{s=1}^{S} K_{h}\left(y-y_{s}(x, \theta)\right) / S$ where $y_{s}(x, \theta)$, $s=1, \ldots ., S$ are i.i.d. draws from $p(\cdot \mid x ; \theta)$. With $\nabla g[d p]$ and $\nabla^{2} g[d p, d p]$ given in eq. (21), we here derive explicit expressions for the terms entering the expansion in eq. (11). First note that this expansion is only valid if Eq. (5) holds. It is easily checked that this is the case with $m=2$ and $\bar{G}_{0}:=E\left[\sup _{\theta \in \Theta}\left\{6\left\|\dot{p}_{i}\left(\theta_{0}\right)\right\| / p_{i}^{3}\left(\theta_{0}\right)+2 / p_{i}^{2}\left(\theta_{0}\right)\right\}\right]$. To ensure $\bar{G}_{0}<\infty$, we either have to assume that the density of covariates is bounded away from zero, or to resort to trimming. Assume for simplicity in the following that the density is bounded away from zero and, moreover, that it is $r$ times differentiable w.r.t. $y$ with its derivatives being integrable, and a $r$ th order kernel is being employed so that $\int z^{i} K(z) d z=0, i=1, \ldots, r-1$ and $\int z^{r} K(z) d z<\infty$ for some $r \geq 2$; finally, $\int K^{\prime}(z)^{2} d z<\infty$ and $\int K^{2}(z) d z<\infty$.

For the analysis of $B_{S, 1}$, note that by standard arguments for kernel estimators, with

$$
\nabla g_{i}(\theta)\left[b_{S}\right]=\frac{h^{r}}{r !} \kappa_{r}\left\{\frac{p_{\theta, i}(\theta)}{p_{i}^{2}(\theta)} \frac{\partial^{r} p_{i}(\theta)}{\partial y_{i}^{r}}-\frac{1}{p_{i}(\theta)} \frac{\partial^{r} \dot{p}_{i}(\theta)}{\partial y_{i}^{r}}\right\}+o\left(h^{r}\right)
$$

and so, we obtain from eq. (9) that

$$
B_{S, 1}=-\frac{\kappa_{r}}{r !} H_{0}^{-1} \frac{h^{r}}{n} \sum_{i=1}^{n} b_{1}\left(y_{i}, x_{i}\right)+o\left(h^{r}\right)=-\frac{\kappa_{r}}{r !} H_{0}^{-1} h^{r} E\left[b_{1}\left(y_{i}, x_{i}\right)\right]+o\left(h^{r}\right),
$$

with $b_{1}\left(y_{i}, x_{i}\right)$ defined in eq. (23). This also implies that

$$
D_{n, S}=\frac{1}{n} \sum_{i=1}^{n} d_{i, S}=-\frac{\kappa_{r}}{r !} \frac{h^{r}}{n} \sum_{i=1}^{n}\left\{b_{1}\left(y_{i}, x_{i}\right)-E\left[b_{1}\left(y_{i}, x_{i}\right)\right]\right\}+o\left(h^{r}\right) .
$$

The above analysis is valid irrespectively of whether a single simulation batch (ECA) or $n$ (EIA) simulation batches are used.

Next, we analyze the variance component $E_{n, S}$. First, consider the EIA: By Lemma 9 , we obtain that $\operatorname{Var}\left(\nabla G_{n}\left[\psi_{S}\right]\right)=O\left(1 /\left(n S h^{d+2}\right)\right)$. More precisely, $E_{n, S}=\nabla G_{n}\left[\psi_{S}\right]=$ $\frac{1}{n} \sum_{i=1}^{n}\left\{a_{S, 1, i}+a_{S, 2, i}\right\}$, where $a_{S, 1, i}$ and $a_{S, 2, i}, i=1, \ldots, n$, are i.i.d. sequences given by, with $y_{i, s}=y_{s}\left(x_{i}, \theta_{0}\right), y_{\theta, i, s}=\partial y_{i, s} /(\partial \theta), p_{i}=p_{i}\left(\theta_{0}\right)$ and so forth

$$
\begin{aligned}
a_{S, 1, i} & =\frac{1}{S} \sum_{s=1}^{S} \frac{p_{\theta, i}}{p_{i}^{2}}\left\{K_{h}\left(y_{i, s}-y_{i}\right)-E_{S}\left[K_{h}\left(y_{i, s}-y_{i}\right)\right]\right\}, \\
a_{S, 2, i} & =\frac{1}{S} \sum_{s=1}^{S} \frac{1}{p_{i}}\left\{K_{h}^{\prime}\left(y_{i, s}-y_{i}\right) y_{\theta, i, s}-E_{S}\left[K_{h}^{\prime}\left(y_{i, s}-y_{i}\right) y_{\theta, i, s}\right]\right\},
\end{aligned}
$$


Applying standard results for kernel regression estimators,

$$
\begin{aligned}
\operatorname{Var}\left(a_{S, 1, i}\right) & =\frac{1}{S} E\left[\frac{\dot{p}_{i} \dot{p}_{i}^{\prime}}{p_{i}^{2}} E\left[\left\{K_{h}\left(y_{i, s}-y_{i}\right)-E_{S}\left[K_{h}\left(y_{i, s}-y_{i}\right)\right]\right\}^{2} \mid y_{i}\right]\right] \\
& =\frac{1}{S h^{d}} E\left[\frac{\dot{p}_{i} \dot{p}_{i}^{\prime}}{p_{i}}\right] \int K(z)^{2} d z, \\
\operatorname{Var}\left(a_{S, 2, i}\right) & =\frac{1}{S} E\left[\frac{1}{p_{i}^{2}} E\left[\left\{K_{h}^{\prime}\left(y_{i, s}-y_{i}\right) \dot{y}_{i, s}-E_{S}\left[K_{h}^{\prime}\left(y_{i, s}-y_{i}\right) \dot{y}_{i, s}\right]\right\}^{2} \mid y_{i}\right]\right] \\
& =\frac{1}{S h^{d+2}} E\left[\frac{\sigma_{\theta, i}^{2}}{p_{i}}\right] \int K^{\prime}(z)^{2} d z .
\end{aligned}
$$

Thus, $E_{n, S}$ has mean zero and variance $\operatorname{Var}\left(E_{n, S}\right) \simeq E\left[\sigma_{\theta, i}^{2} / p_{i}\right] \int K^{\prime}(z)^{2} d z /\left(n S h^{d+2}\right)$. In the case of ECA, Kristensen and Shin (2012) showed that $\operatorname{Var}\left(E_{n, S}\right)=\operatorname{Var}\left(\nabla G\left[e_{s}\right]\right) / S+$ $O\left(1 /\left(n S h^{d+1}\right)\right)$.

Finally, consider $B_{S, 2}$ : First note that

$$
\nabla^{2} g_{i}\left[\psi_{S}, \psi_{S}\right]=\frac{2}{p_{i}^{2}}\left\{\frac{\partial \hat{p}_{i, S}}{\partial \theta}-E\left[\frac{\partial \hat{p}_{i, S}}{\partial \theta}\right]\right\}\left\{\hat{p}_{i, S}-E_{S}\left[\hat{p}_{i, S}\right]\right\}-\frac{2 p_{\theta, i}}{p_{i}^{3}}\left\{\hat{p}_{i, S}-E_{S}\left[\hat{p}_{i, S}\right]\right\}^{2}
$$

With $m\left(x, \varepsilon_{s}\right)=y_{s}\left(x, \theta_{0}\right)$ and $\varepsilon_{s}$ being i.i.d. draws from some density $f_{\varepsilon}(\varepsilon)$ we obtain $p(y \mid x)=p\left(y \mid x ; \theta_{0}\right)=f_{\varepsilon}(r(x, y))\left|r_{y}(x, y)\right|$, where $r(x, y)$ denotes the inverse of $m(x, \varepsilon)$ so that $\varepsilon=r(x, y)$. Then, for both ECA and EIA, with " $\simeq$ " indicating that only leading terms are included,

$$
\begin{aligned}
& E_{S}\left[\left\{\frac{\partial \hat{p}_{i, S}}{\partial \theta}-E\left[\frac{\partial \hat{p}_{i, S}}{\partial \theta}\right]\right\}\left\{\hat{p}_{i, S}-E_{S}\left[\hat{p}_{i, S}\right]\right\}\right] \\
\simeq & \frac{1}{S} E_{S}\left[K_{h}^{\prime}\left(m\left(x_{i}, \varepsilon_{s}\right)-y_{i}\right) K_{h}\left(m\left(x_{i}, \varepsilon_{s}\right)-y_{i}\right) \dot{m}\left(x_{i}, \varepsilon_{s}\right)\right] \\
= & \frac{1}{S} \int K_{h}^{\prime}\left(y-y_{i}\right) K_{h}\left(y-y_{i}\right) \dot{m}\left(x_{i}, r\left(x_{i}, y\right)\right) p\left(y \mid x_{i}\right) d y \\
= & \frac{1}{S h^{d+1}} \int K(z) K^{\prime}(z) d z \times \dot{m}\left(x_{i}, r\left(x_{i}, y_{i}\right)\right) p\left(y_{i} \mid x_{i}\right),
\end{aligned}
$$

while, by similar arguments,

$$
E_{S}\left[\left\{\hat{p}_{i, S}-E_{S}\left[\hat{p}_{i, S}\right]\right\}^{2}\right] \simeq \frac{1}{S} E\left[K_{h}^{2}\left(m\left(x_{i}, \varepsilon_{s}\right)-y_{i}\right)\right]=\frac{1}{S h^{d}} \int K^{2}(z) d z \times p_{i} .
$$

Substituting the resulting expression of $E\left[\nabla^{2} g_{i}\left[\psi_{S}, \psi_{S}\right]\right]$ into eq. (9), we obtain the claimed expression in eq. (22). 


\section{C.2 Newton-Raphson in empirical IO}

For notational simplicity, we drop the market subscript $i$ from the notation-calculations are done separately on each market until the last summation. We start with the identity $\gamma\left(x, \gamma^{-1}(x, m ; \theta) ; \theta\right) \equiv m$ to obtain

$$
\frac{\partial \gamma}{\partial \xi}\left(x, \gamma^{-1}(x, m ; \theta) ; \theta\right) \frac{\partial \gamma^{-1}}{\partial \theta}(x, m ; \theta)+\frac{\partial \gamma}{\partial \theta}\left(x, \gamma^{-1}(x, m ; \theta) ; \theta\right)=0
$$

and therefore

$$
\frac{\partial \gamma^{-1}}{\partial \theta}(x, m ; \theta)=-\left[\frac{\partial \gamma}{\partial \xi}\left(x, \gamma^{-1}(x, m ; \theta) ; \theta\right)\right]^{-1} \frac{\partial \gamma}{\partial \theta}\left(x, \gamma^{-1}(x, m ; \theta) ; \theta\right)
$$

To find expressions for the two functions on the right-hand side of the last equation, define $Q_{j}(R)=\exp \left(R_{j}\right) /\left(1+\sum_{k=1}^{J} \exp \left(R_{k}\right)\right)$. Its differential is

$$
\frac{d Q_{j}}{Q_{j}}=d R_{j}-d \log \left(1+\sum_{l=1}^{J} \exp \left(R_{l}\right)\right)=d R_{j}-\sum_{l=1}^{J} Q_{l} d R_{l} .
$$

In particular, $\partial \nu_{j, s} /\left(\partial \xi_{l}\right)=\nu_{j, s}\left(\delta_{j l}-\nu_{l, s}\right)$, where $\delta_{j l}$ is the Kronecker symbol $\mathbb{1}(j=l)$. Therefore

$$
\frac{\partial \hat{\gamma}_{S, j}}{\partial \xi_{l}}(x, \xi ; \theta)=\hat{\gamma}_{S, j}(x, \xi ; \theta) \delta_{j l}-\frac{1}{S^{*}} \sum_{s=1}^{S^{*}} \nu_{j, s}(x, \xi, \varepsilon ; \theta) \nu_{l, s}(x, \xi, \varepsilon ; \theta)
$$

which can be rewritten as

$$
\frac{\partial \hat{\gamma}_{S}}{\partial \xi}(x, \xi ; \theta)=\frac{1}{S^{*}} \sum_{s=1}^{S^{*}}\left(\operatorname{diag}\left(\nu_{s}(x, \xi, \varepsilon ; \theta)\right)-\left(\nu_{s}(x, \xi, \varepsilon ; \theta) \otimes \nu_{s}(x, \xi, \varepsilon ; \theta)\right)\right)
$$

where $\operatorname{diag}(a)$ is the square matrix with the vector $a$ on the diagonal, and $\otimes$ is the Kronecker (outer) product.

To compute derivatives with respect to $\theta$, we start with another application of (34):

$$
\begin{aligned}
\frac{\partial \nu_{j, s t}}{\partial \beta_{q}}(\xi ; \theta) & =\nu_{j, s}(x, \xi, \varepsilon ; \theta)\left(x_{j, q}-\sum_{l=1}^{J} \nu_{l, s}(x, \xi, \varepsilon ; \theta) x_{l, q}\right) \\
\frac{\partial \nu_{j, s}}{\partial a_{r}}(\xi, \theta) & =\nu_{j, s}(x, \xi, \varepsilon ; \theta) \varepsilon_{s}\left(x_{j, q}-\sum_{l=1}^{J} \nu_{l, s}(x, \xi, \varepsilon ; \theta) x_{l, q}\right) .
\end{aligned}
$$

Now denote, for any matrix with $J$ rows $\left(X_{j}\right)_{j=1}^{J}, \hat{X}_{j, s}=X_{j}-\sum_{k=1}^{J} \nu_{k, s}(x, \xi, \varepsilon ; \theta) X_{k}$. It 
follows that taking $X$ to be the matrix $\left(x_{j q}\right)$,

$$
\frac{\partial \hat{\gamma}_{j}}{\partial \beta}(x, \xi ; \theta)=\frac{1}{S^{*}} \sum_{s=1}^{S^{*}} \nu_{j, s}(x, \xi, \varepsilon ; \theta) \hat{x}_{j, s}, \quad \frac{\partial \hat{\gamma}_{j}}{\partial A}(x, \xi ; \theta)=\frac{1}{S^{*}} \sum_{s=1}^{S^{*}} \nu_{j, s}(x, \xi, \varepsilon ; \theta)\left(\hat{x}_{j, s} \otimes \varepsilon_{s}\right) .
$$

This gives all elements of the procedure delineated in section 6.2 .

\section{Expansion with multiple approximators}

We here generalize the theory to handle the case where multiple approximation methods are employed. Let $\hat{\theta}_{n}$ satisfy a first order condition of the form

$$
G_{n}\left(\hat{\theta}_{n}, \gamma_{0,1}, \ldots, \gamma_{0, M}\right)=o_{P}(1 / \sqrt{n})
$$

for some random functional $G_{n}\left(\theta, \gamma_{1}, \ldots, \gamma_{M}\right)$. The corresponding approximate estimator $\hat{\theta}_{n, S}$ satisfies

$$
G_{n}\left(\hat{\theta}_{n, S}, \hat{\gamma}_{S_{1}, 1}, \ldots, \hat{\gamma}_{S_{M}, M}\right)=o_{P}(1 / \sqrt{n})
$$

Here, we allow for $\gamma_{m}, m=1, \ldots, M$, being approximated using different methods and with different degrees of approximations, $S_{m}, m=1, \ldots, M$, which we collect in $S=\left(S_{1}, \ldots, S_{M}\right)$. Collect the approximated functions in $\gamma=\left(\gamma_{1}, \ldots . \gamma_{M}\right)$ and assume that $G_{n}(\theta, \gamma)$ takes the form of a sample average, $G_{n}(\theta, \gamma)=\frac{1}{n} \sum_{i=1}^{n} g\left(z_{i} ; \theta, \gamma\right)$. We assume that $\gamma_{0, m}$ belongs to a linear function space $\Gamma_{m}$ equipped with a norm $\|\cdot\|_{m}, m=1, \ldots, M$, so that $\gamma \in \Gamma=$ $\Gamma_{1} \times \cdots \Gamma_{M}$ with norm $\|\gamma\|=\sum_{m=1}^{M}\left\|\gamma_{m}\right\|$. We maintain the same notation as in the case of one function being approximated and, for example, let $H_{n}(\theta, \gamma)=\frac{1}{n} \sum_{i=1}^{n} h\left(z_{i} ; \theta, \gamma\right)$, with $h\left(z_{i} ; \theta, \gamma\right)=\partial g\left(z_{i} ; \theta, \gamma\right) /(\partial \theta)$, denote the first-order derivative of the sample moments. With the same notation, Assumptions A.1-A.3 provided in the main text remain unchanged. Next, we generalize Assumptions A.4-A.5 to:

A.4* $(m)$ Assumption A.4 holds with $\nabla g(z ; \theta)[d \gamma]=\sum_{k=1}^{M} \nabla_{m} g(z ; \theta)\left[d \gamma_{k}\right]$ and $\nabla^{2} g(z ; \theta)[d \gamma, d \gamma]=$ $\sum_{j, k=1}^{M} \nabla_{j, k}^{2} g(z ; \theta)\left[d \gamma_{j}, d \gamma_{k}\right]$

A.5* $(p)$ For $m=1, \ldots, M$ : The approximator $\hat{\gamma}_{S, m}$ lies in $\Gamma_{m}$ and satisfies:

(ii) Its bias $b_{S, m}(z ; \theta)=E\left[\hat{\gamma}_{S, m}(x ; \theta)\right]-\gamma_{0, m}(x ; \theta)$ is of order $\beta_{m}>0$ :

$$
b_{S, m}(x ; \theta)=S^{-\beta_{m}} \bar{b}_{m}(x ; \theta)+o\left(S^{-\beta_{m}}\right) .
$$

(iii) For some $p \geq 2$ and all $2 \leq q \leq p$, there exists $\alpha_{m, q}>0$ so that $\psi_{S, m}(x ; \theta)=$ 


$$
\begin{aligned}
\hat{\gamma}_{S, m}(x ; \theta)-E\left[\hat{\gamma}_{S, m}(x ; \theta)\right] \text { satisfies: } & \\
& E\left[\left\|\psi_{S, m}(x ; \theta)\right\|^{q}\right]=S^{-\alpha_{m, q}} v_{m, q}(x ; \theta)+o\left(S^{-\alpha_{m, q}}\right) .
\end{aligned}
$$

The leading bias and variance terms take the form

$$
\begin{aligned}
B_{S, 1}=-H_{0}^{-1} \sum_{m=1}^{M} E\left[\nabla_{m} g_{i}\left[b_{S_{m}, m}\right]\right], \quad B_{S, 2}=-\frac{1}{2} H_{0}^{-1} \sum_{k, m=1}^{M} E\left[\nabla_{k, m}^{2} g_{i}\left[\psi_{S_{k}, k}, \psi_{S_{m}, m}\right]\right] \\
D_{n, S}=\frac{1}{n} \sum_{i=1}^{n} d_{i, S}, \quad d_{i, S}=\sum_{m=1}^{M}\left\{\nabla_{m} g_{i}\left[b_{S_{m}, m}\right]-E\left[\nabla_{m} g_{i}\left[b_{S_{m}, m}\right]\right]\right\} \\
E_{n, S}=\frac{1}{n} \sum_{i=1}^{n} \sum_{m=1}^{M} \nabla_{m} g_{i}\left[\psi_{S_{m}, m}\right] .
\end{aligned}
$$

We now obtain the following expansion of the approximate estimator, which generalizes the "univariate" version.

Theorem 13 Assume A.1-A.3, A.4*(2), and A.5*(4). Then,

$\hat{\theta}_{n, S}-\theta_{0}=B_{S, 1}+B_{S, 2}+H_{0}^{-1}\left\{G_{n}+D_{n, S}+E_{n, S}\right\}+O_{P}\left(\sum_{m=1}^{M}\left\{S_{m}^{-3 \beta_{m}}+S_{m}^{-\alpha_{m, 3}}\right\}\right)+o_{P}(1 / \sqrt{n})$,

where $G_{n}=G_{n}\left(\theta_{0}, \gamma_{0}\right)$ and the two sequences $\left(G_{n}, D_{n, S}\right)$ and $E_{n, S}$ are asymptotically mutually independent. Moreover, the following limit results hold as $n, S \rightarrow \infty$ :

- $\sqrt{n}\left(\Omega_{S}^{G+D}\right)^{1 / 2}\left\{G_{n}+D_{n, S}\right\} \stackrel{d}{\rightarrow} N\left(0, I_{k}\right)$ with $\Omega^{G}=\sum_{i=-\infty}^{\infty} \operatorname{Cov}\left(g_{0}, g_{i}\right)$ and

$$
\Omega_{S}^{G+D}=\sum_{i=-\infty}^{\infty} \operatorname{Cov}\left(g_{0}+d_{0, S}, g_{i}+d_{i, S}\right)=\Omega^{G}+O\left(\sum_{m=1}^{M} S_{m}^{-2 \beta_{m}}\right) .
$$

- The bias terms have orders $B_{S, 1}=O\left(\sum_{m=1}^{M} S_{m}^{-\beta_{m}}\right)$ and $B_{S, 2}=O\left(\sum_{k, m=1}^{M} \sqrt{S_{k}^{-\alpha_{k, 2}} S_{m}^{-\alpha_{m, 2}}}\right)$.

- $\operatorname{Var}\left(E_{n, S}\right)=O_{P}\left(\sum_{m=1}^{M} S_{m}^{-\alpha_{m, 2}} / n\right)(E I A)$ or $\operatorname{Var}\left(E_{n, S}\right)=O_{P}\left(\sum_{m=1}^{M} S_{m}^{-\alpha_{m, 2}}\right)$ (ECA).

- If in addition Assumption A.6*(4) holds with $w_{S, m} \equiv w_{m}$ not depending on $S$, then 


$$
\begin{gathered}
\alpha_{m, 2}=1, m=1, \ldots, M \text {, and } \\
\text { EIA }:\left\{\frac{1}{\sqrt{n S_{m}}} \sum_{i=1}^{n} \nabla_{m} g_{i}\left[\psi_{S_{m}, m, i}\right]\right\}_{m=1}^{M} \rightarrow^{d} N\left(0, \Omega_{\mathrm{EIA}}^{E}\right), \\
\text { EIA }:\left\{\frac{1}{\sqrt{S_{m}}} \sum_{i=1}^{n} \nabla_{m} g_{i}\left[\psi_{S_{m}, m}\right]\right\}_{m=1}^{M} \rightarrow^{d} N\left(0, \Omega_{\mathrm{ECA}}^{E}\right) \\
\text { where } \Omega_{\mathrm{EIA}}^{E}=\left[\Omega_{\mathrm{EIA}, k m}^{E}\right]_{k, m=1}^{M} \text { and } \Omega_{\mathrm{ECA}}^{E}=\left[\Omega_{\mathrm{ECA}, k m}^{E}\right]_{k, m=1}^{M} w i t h \\
\Omega_{\mathrm{EIA}, k m}^{E}=\lim _{S \rightarrow \infty} \frac{1}{\sqrt{S_{k} S_{m}}} \operatorname{Cov}\left(\sum_{s=1}^{S_{k}} \nabla_{k} g_{0}\left[e_{k, s}\right], \sum_{s=1}^{S_{m}} \nabla_{m} g_{0}\left[e_{m, s}\right]\right), \\
\Omega_{\mathrm{ECA}, k m}^{E}=\lim _{S \rightarrow \infty} \frac{1}{\sqrt{S_{k} S_{m}}} \operatorname{Cov}\left(\sum_{s=1}^{S_{k}} \nabla_{k} G\left[\tilde{e}_{k, s}\right], \sum_{s=1}^{S_{m}} \nabla_{m} G\left[\tilde{e}_{m, s}\right]\right) .
\end{gathered}
$$

If the approximators are mutually independent, the second bias component simplifies to

$$
B_{S, 2}=-\frac{1}{2} H_{0}^{-1} \sum_{m=1}^{M} E\left[\nabla_{m, m}^{2} g_{i}\left[\psi_{S_{m}, m}, \psi_{S_{m}, m}\right]\right]=O_{P}\left(\sum_{m=1}^{M} S_{m}^{-\alpha_{m, 2}}\right),
$$

and the off-diagonal elements of the covariance matrices $\Omega_{\mathrm{EIA}}^{E}$ and $\Omega_{\mathrm{ECA}}^{E}$ become zero.

Proof. By Lemma 7,

$$
\hat{\theta}_{n, S}-\hat{\theta}_{n}=-H_{0}^{-1}\left\{G_{n}\left(\theta_{0}, \hat{\gamma}_{S}\right)-G_{n}\left(\theta_{0}, \gamma_{0}\right)\right\}+o_{P}(1 / \sqrt{n})
$$

The expansion $(m=2)$ given in eq. (6) then yields

$$
\left\|\hat{\theta}_{n, S}-\hat{\theta}_{n}\right\|=O_{P}\left(\left\|\nabla G_{n}\left(\theta_{0}\right)\left[\Delta \hat{\gamma}_{S}\right]+\frac{1}{2} \nabla^{2} G_{n}\left(\theta_{0}\right)\left[\Delta \hat{\gamma}_{S}, \Delta \hat{\gamma}_{S}\right]+R_{n, S}\right\|\right)+o_{P}(1 / \sqrt{n}),
$$

where $\nabla G_{n}\left(\theta_{0}\right)[d \gamma]=\sum_{m=1}^{M} \nabla_{m} G_{n}\left(\theta_{0}\right)\left[d \gamma_{m}\right], \nabla^{2} G_{n}\left(\theta_{0}\right)[d \gamma, \Delta \gamma]=\sum_{k, m=1}^{M} \nabla_{k, m}^{2} G_{n}\left(\theta_{0}\right)\left[d \gamma_{k}, d \gamma_{m}\right]$, and $\Delta \hat{\gamma}_{m, S_{m}}=\hat{\gamma}_{m, S_{m}}-\gamma_{m, 0}$. The rate of the remainder term $R_{n, S}$ follows by the same arguments as before, $E\left[\left\|R_{n, S}\right\|\right] \leq \frac{\bar{G}_{0}}{n} \sum_{i=1}^{n} E\left[\left\|\Delta \hat{\gamma}_{S}\right\|^{3}\right]$, where

$$
E\left[\left\|\Delta \hat{\gamma}_{S}\right\|^{3}\right] \leq 4 \sum_{m=1}^{M}\left\{E\left[\left\|\psi_{S_{m}, m}\right\|^{3}\right]+4 E\left[\left\|b_{S_{m}, m}\right\|^{3}\right]\right\}=O\left(\sum_{m=1}^{M}\left\{S_{m}^{-\alpha_{m, 3}}+S_{m}^{-3 \beta_{m}}\right\}\right)
$$

The rate of $\nabla_{m} G_{n}\left(\theta_{0}\right)\left[\Delta \hat{\gamma}_{S_{m}, m}\right]$ follow directly from Lemma 10 while $\nabla_{k, m}^{2} G_{n}\left(\theta_{0}\right)\left[\Delta \hat{\gamma}_{S_{k}, k}, \Delta \hat{\gamma}_{S_{m}, m}\right]$ is analyzed by a simple extension of the arguments employed in the single-approximator case. More specifically, Lemma 9 still applies and yields $\operatorname{Var}\left(\nabla_{k, m}^{2} G_{n}\left(\theta_{0}\right)\left[b_{S_{k}, k}, b_{S_{m}, m}\right]\right)=$ 
$O\left(n^{-1} S_{k}^{-\beta_{k}} S_{m}^{-\beta_{m}}\right)$, for $k, m=1, \ldots, M$. To bound the variance component, apply Lemma 9 to obtain that $\left.E\left[\| \nabla_{k, m}^{2} G\left(\theta_{0}\right)\left[\psi_{S_{k}, k}, \psi_{S_{m}, m}\right]\right] \| \mid\right]=O_{P}\left(S_{k}^{-\alpha_{k, 2}} S_{m}^{-\alpha_{m, 2}}\right), E\left[\nabla_{k, m}^{2} G_{n}\left[\psi_{S_{m}, m}, b_{S_{k}, k}\right]\right]=$ 0 while $\operatorname{Var}\left(\nabla^{2} G_{n}\left[\psi_{S_{k}, k}, b_{S_{m}, m}\right]\right)=O\left(n^{-1} S_{k}^{-\alpha_{k, 2}} S_{m}^{-2 \beta_{m}}\right)$, for $k, m=1, \ldots, M$. The weak convergence results follow by the same arguments as in the proof of Theorem 2.

The analytical bias adjustments proposed in Section 5 straightforwardly generalize to the above set-up by simply setting

$$
\begin{aligned}
\nabla^{2} g\left(z_{i} ; \theta, \hat{\gamma}_{S}\right)\left[\hat{\psi}_{i, S}, \hat{\psi}_{i, S}\right] & =\sum_{k, m=1}^{M} \nabla_{k, m}^{2} g\left(z_{i} ; \theta, \hat{\gamma}_{S}\right)\left[\hat{\psi}_{i, S_{k}, k}, \hat{\psi}_{i, S_{m}, m}\right] \\
\nabla^{2} g\left(z_{i} ; \theta, \hat{\gamma}_{S}\right)\left[\hat{e}_{i, s, S}, \hat{e}_{i, s, S}\right] & =\sum_{k, m=1}^{M} \nabla_{k, m}^{2} g\left(z_{i} ; \theta, \hat{\gamma}_{S}\right)\left[\hat{e}_{i, s, S_{k}, k}, \hat{e}_{i, s, S_{m}, m}\right]
\end{aligned}
$$

in eqs. (16) and (17), respectively. If the $M$ approximators are mutually independent, the cross terms in the above double sums can be left out. The adjustments of the standard errors also remain valid when using the definitions of $\nabla g\left(z ; \theta_{0}\right)[d \gamma]$ and $\nabla^{2} g\left(z ; \theta_{0}\right)[d \gamma, d \gamma]$ given in A.4* Finally, the Newton-Raphson procedure will still work with $S^{*}=\left(S_{1}^{*}, \ldots, S_{M}^{*}\right)$ where $S_{m}^{*}>S_{m}, m=1, \ldots, M$. 